\title{
1 Genome architecture shapes evolutionary adaptation to DNA replication stress
}

2

\author{
Marco Fumasoni*, Andrew W. Murray*
}

Department of Molecular and Cellular Biology, Harvard University, Cambridge, United States

\section{ABSTRACT}

In haploid budding yeast, evolutionary adaptation to constitutive DNA replication stress alters three genome maintenance modules: DNA replication, the DNA damage checkpoint, and sister chromatid cohesion. We asked how these trajectories depend on a population's genome architecture by comparing the adaptation of haploids to that diploids and recombination deficient haploids. In all three genome architectures, adaptation happens within 1000 generations at rates that are linearly correlated with the initial fitness defect of the ancestors. Mutations in individual genes are selected at different frequencies in different architectures, but the benefits these mutations confer are similar in all three architectures, and combinations of these mutations reproduce the fitness gains of evolved populations. Despite the differences in the selected mutations, the evolutionary trajectories target the same three functional modules in all architectures revealing a common evolutionary response to DNA replication stress.

*For correspondence: marcofumasoni@fas.harvard.edu, awm@mcb.harvard.edu 


\section{INTRODUCTION}

20 Organisms and cells adapt to selective pressures through the acquisition of beneficial mutations.

21 The interactions between beneficial mutations that produce evolved phenotypes are complex and difficult to determine both in laboratory and natural evolution. These interactions are also likely to depend on two factors. The first, genetic background, refers to the collection of individual sequence variants in a strain or population [1-4]. The second is genome architecture, which we use to refer to functional features of the genome including its ploidy, rates of recombination, and the activity of mobile genetic elements. Previous work has revealed that different genetic backgrounds can select different spectra of mutations and different genome architectures lead to different rates of adaptation [5-16] but we lack a general, mechanistic explanation of these differences. Here, we take advantage of our earlier work on adaptation to DNA replication stress to investigate how different genome architectures control adaptive evolutionary trajectories.

We previously characterized the evolutionary adaptation of haploid cells of the budding yeast, Saccharomyces cerevisiae, experiencing a form of constitutive DNA replication stress [17], a perturbation of DNA synthesis that produces lesions in DNA, increases genome instability, and decreases fitness [18-21]. DNA replication stress is often present in cancer cells as a consequence of oncogene activation [22-25] and can also be generated by the unrestrained proliferation of selfish genetic elements such as viruses or transposons [26-29]. In haploid $S$. cerevisiae, evolutionary adaptation to constitutive DNA replication stress is driven by mutations affecting three different functional modules that contribute to genome maintenance: gain-of-function mutations that stabilize the linkage between sister chromatids, mutations that improve the completion of DNA replication, most likely by stabilizing the progression of replication forks, and mutations that inactivate the DNA damage checkpoint, thus accelerating the cell division cycle. Full adaptation to the defects imposed by DNA replication stress requires a combination of mutations in all three modules, which are acquired in an order dictated by the epistatic interactions between the mutations [17].

We asked how genome architecture affected evolutionary adaptation to constitutive DNA replication stress by testing two additional architectures: i) diploid cells, which carry two copies of every chromosome and thus make fully recessive mutations phenotypically silent, ii) haploid, recombination-deficient cells which cannot produce gene amplifications or deletions that depend on homologous recombination between repeated sequences [30,31]. These architectures are relevant for cancer biology: cancer progression begins in diploid cells and defects in homologous recombination have been implicated in predisposition to tumorigenesis (i.e. BRCA2 mutations, [32,33]) and exploited for anticancer therapies [34-36].

We evolved cells with these two genome architectures and compared their responses with those of haploid, recombination-proficient cells. All three architectures experienced constitutive DNA replication stress caused by removing Ctf4 [17], a protein that physically links different activities at replication forks [37]. Adaptation to DNA replication stress occurs in all three genome 
architectures at rates that are correlated with their initial fitness defects. Whole genome sequencing of the evolved populations revealed diverse mutational spectra with only modest convergence among the three different architectures. Despite the differences in which genes were mutated, all three architectures accumulated mutations in the DNA replication machinery, the DNA damage checkpoint, and sister chromatid cohesion. Individually engineering a subset of these mutations into all three architectures demonstrated their ability to reduce the fitness cost of DNA replication stress, even in architectures where they were not selected. Combining mutations in all three modules produced fitness increases that matched those of the evolved strains in all three architectures. We conclude that although differences in the observed frequency of mutations in different genes depend on genome architecture, the principles that govern adaptation to constitutive DNA replications stress are largely architecture-independent. We discuss the implication of our results for tumor evolution and for generating inter-species molecular diversity in genome maintenance.

\section{RESULTS}

All populations adapt to constitutive DNA replication stress.

DNA replication stress is often induced with drugs or by reducing the level of DNA polymerases [38-41]. To avoid evolving drug resistance or increased polymerase expression, which would rapidly overcome DNA replication stress, we deleted the CTF4 gene, which encodes a nonessential subunit of the DNA replication machinery (the replisome) [42]. Ctf4 is a homo-trimer that functions as a structural hub within the replisome $[37,43]$ by binding to the replicative DNA helicase, primase (the enzyme that makes the RNA primers that initiate DNA replication), and other accessory factors [37,44-46]. In the absence of $\mathrm{Ctf} 4$, the Pol $\alpha$-primase and other lagging strand processing factors are poorly recruited to the replisome $[37,44,47]$, causing several characteristic features of DNA replication stress, such as accumulation of single strand DNA (ssDNA) gaps [48,49], reversed and stalled forks [17,49], cell cycle checkpoint activation $[47,50]$ and altered chromosome metabolism [51,52]. As a consequence of these defects, ctf $4 \Delta$ cells have substantially reduced reproductive fitness [17]. We removed CTF4 from wild-type (WT) haploid and diploid cells, as well as from haploids impaired in homologous recombination due to the deletion of RAD52 (Figure 1A), which encodes a conserved enzyme required for pairing homologous DNA sequences during recombination [53]. Because Rad52 is involved in different forms of homologous recombination, it's absence produces the most severe recombination defects and thus allows us to achieve the largest recombination defect achievable with a single gene deletion [54]. Attempts to generate homozygous ctf4 $\Delta / \mathrm{ctf} 4 \Delta \mathrm{rad} 52 \Delta / \mathrm{rad} 52 \Delta$ mutants failed due to the lethality of the resulting zygotes. We refer to the strains we analyzed as haploids (ctf4 $\Delta$, yellow), diploids (ctf $4 \Delta / c t f 4 \Delta$, blue) and recombination-deficient (ctf $4 \Delta$ rad52 $\Delta$, red). Compared to the previously reported ctf $4 \Delta$ haploid strains, which manifested a fitness decrease 
absence of Ctf4 was more detrimental in diploid ( $35 \%$ reduced fitness) and recombinationdeficient strains ( $\sim 55 \%$ reduced fitness) (Figure $1 B$ ). Diploid cells require twice as many forks as haploids and Ctf4-deficient diploids are thus more likely to have forks that cause severe cell-cycle delays or cell lethality. We speculate that this increased probability explains the more prominent fitness defect displayed by diploid cells. Interestingly, homologs of Ctf4 are absent in prokaryotes, where the primase is physically linked to the replicative helicase [55] and Ctf4 is essential in the cells of eukaryotes with larger genomes such as chickens [48] and humans [56]. Rad52 is likely involved in rescuing stalled replication forks by recombination-dependent mechanisms $[49,57]$. We speculate that the absence of Rad52 increases the duration of these stalls and leads some of them to become double-stranded breaks resulting in cell lethality and explaining the decreased fitness of $c t f 4 \Delta$ rad52 $\Delta$ haploid double mutants. In diploids ctf4 $\Delta$ rad52 $\Delta$ cells, which have twice as many chromosomes, the number of irreparably stalled fork may be sufficient to kill most of the cells in a population, thus explaining the unviability of the strain.

109

110

111

112

113

114

115

116

117

118

119

120

121

122

123

124

125

126

127

128

129

We evolved eight parallel diploid and recombination-deficient populations for 1000 generations in the same conditions and at the same time as the haploid populations we previously analyzed ([17] and see Materials and methods). In this regime, spontaneous mutations that increase fitness and survive genetic drift will spread through the population [58,59]. In addition, we evolved eight wild-type diploid populations like the previously evolved wild-type haploids, to control for mutations that are selected by the culture conditions rather than by ameliorating DNA replication stress [17].

After 1000 generations experiencing constitutive DNA replication stress, we asked how the fitness of the evolved diploid and recombination-deficient populations compared to those of evolved haploids. Regardless of their genome architecture, all populations lacking Ctf4 increased in fitness compared to their respective ancestors: haploids from $-27 \%$ to $-9.6 \pm 1.2 \%$ [17], diploids from $-35 \%$ to $-14.4 \pm 1.8 \%$, and recombination-deficient haploids from $-55 \%$ to $-19 \pm 1 \%$ (Figure 1B). The fitness of four clones isolated from each evolved population were similar to the fitness of the population they were derived from (Figure 1-S1). One diploid line (EVO14) gave rise to a population with a haploid genome content, suggesting a possible haploidization event during evolution. Sequencing revealed no aneuploidies as a potential explanation of this phenomenon. While diploidization has been recurrently observed during experimental evolution with budding yeast [8,60-62], reports of spontaneous haploidization events have been instead scarce. Given the difficulties introduced by the change of ploidy over the 1000 generations, we have excluded EVO14 from all our analyses. Control evolved wild-type populations showed only small fitness increases: $4 \pm 1 \%$ for haploids and $6.7 \pm 2.7 \%$ for diploids (Figure $1-S 2$ ). 
A

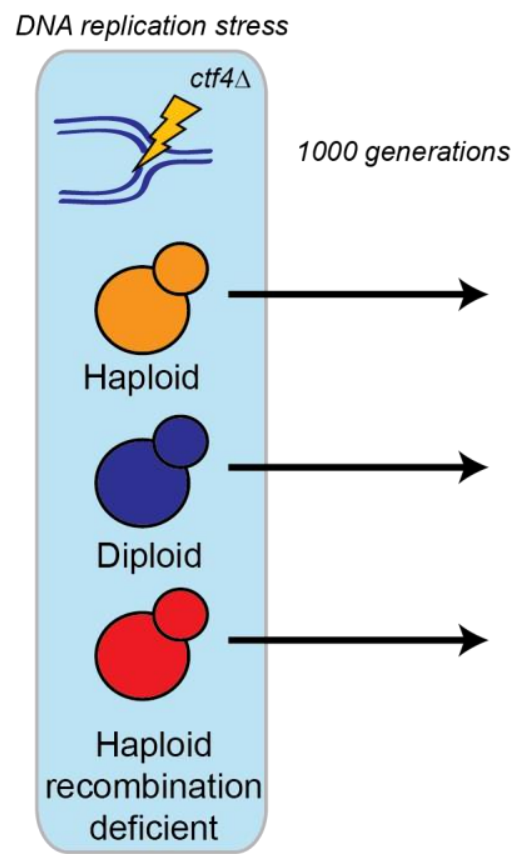

B

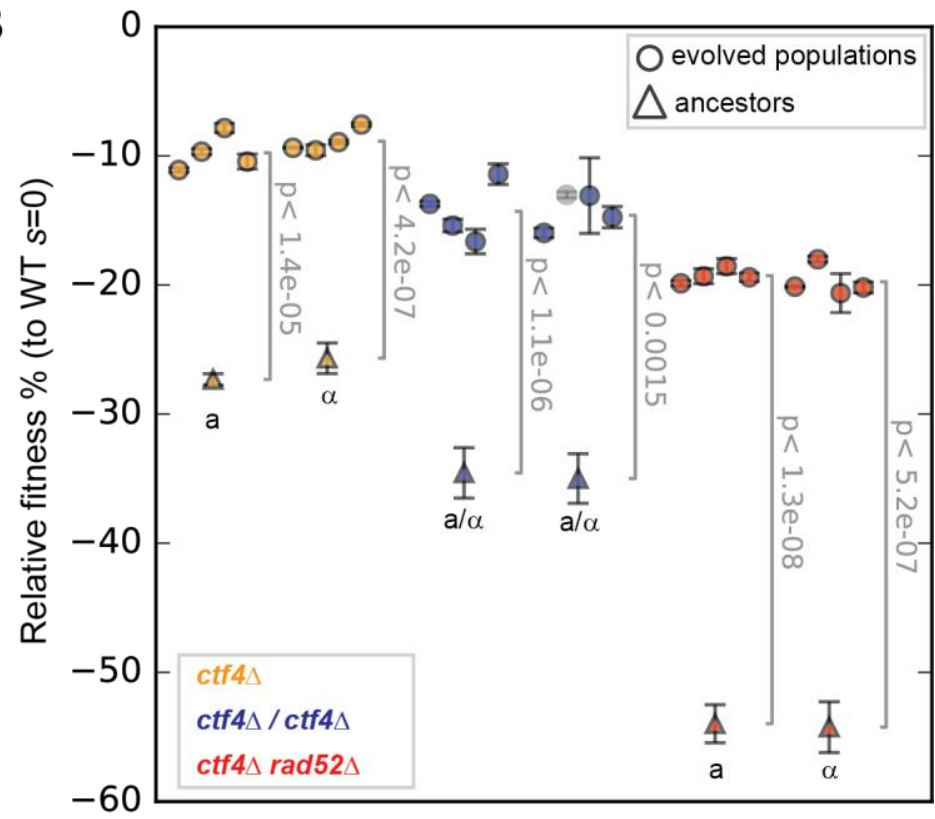

Figure 1: Evolutionary adaptation to constitutive DNA replication stress in different genome architectures. (A) Experimental scheme: Constitutive DNA replication stress was induced by removing $\mathrm{Ctf} 4$, a protein that coordinates replisome activities. Haploid (orange), diploids (blue) and cells with a severely decreased ability to perform homologous recombination (red, recombination-deficient), were subjected to 1000 generations of experimental evolution in the presence of constitutive DNA replication stress. (B) The fitness of the 6 ancestral strains ( 2 for each genome architecture) and of 24 evolved populations derived from them, relative to WT cells $(s=0)$. Cells were competed against reference strains with the same ploidy. Fitness data of haploid strains (orange) is from [17]. The semi-transparent gray dot represents a diploid strain that became haploid during the experiment and is thus excluded from subsequent analysis. Error bars represent standard deviations. a and $\alpha$ refer to the strains' mating type (MAT locus). a/ $\alpha$ indicates diploid strains. The P-values reported in figures are the result of $t$-tests assuming unequal variances (Welch's test). The fitness values shown here are reported in Source data 1.

\section{The rate of adaptation to DNA replication stress depends on the ancestor's fitness}

Although all populations increased their fitness in response to constitutive DNA replication stress, strains with different genome architectures increased in fitness to different extents (measured as the absolute fitness gain of populations over 1000 generations). Recombination-deficient populations showed the largest fitness gain $(+34.6 \pm 0.8 \%)$, followed by diploid $(+20.3 \pm 1.6 \%)$ and haploid populations ( $+17.2 \pm 1.0 \%$, Figure $2 \mathrm{~A})$. We asked what could have generated different adaptation rates in response to the same selective pressures. Less fit ancestral strains have been proposed to increase in fitness faster than more fit ancestors, a phenomenon referred as 'declining adaptability' [63-66]. To test for this possibility, we plotted the final fitness of all evolved populations against the fitness of their respective ancestors (Figure $2 \mathrm{C}$ ). We found that the absolute fitness gain over 1000 generations depended linearly on the ancestors' relative 
154 fitness $\left(R^{2}=0.95\right)$. These results agree with previous studies, which observed declining 155 adaptability across a wide range of organisms and selection conditions $[11,12,67,68]$.
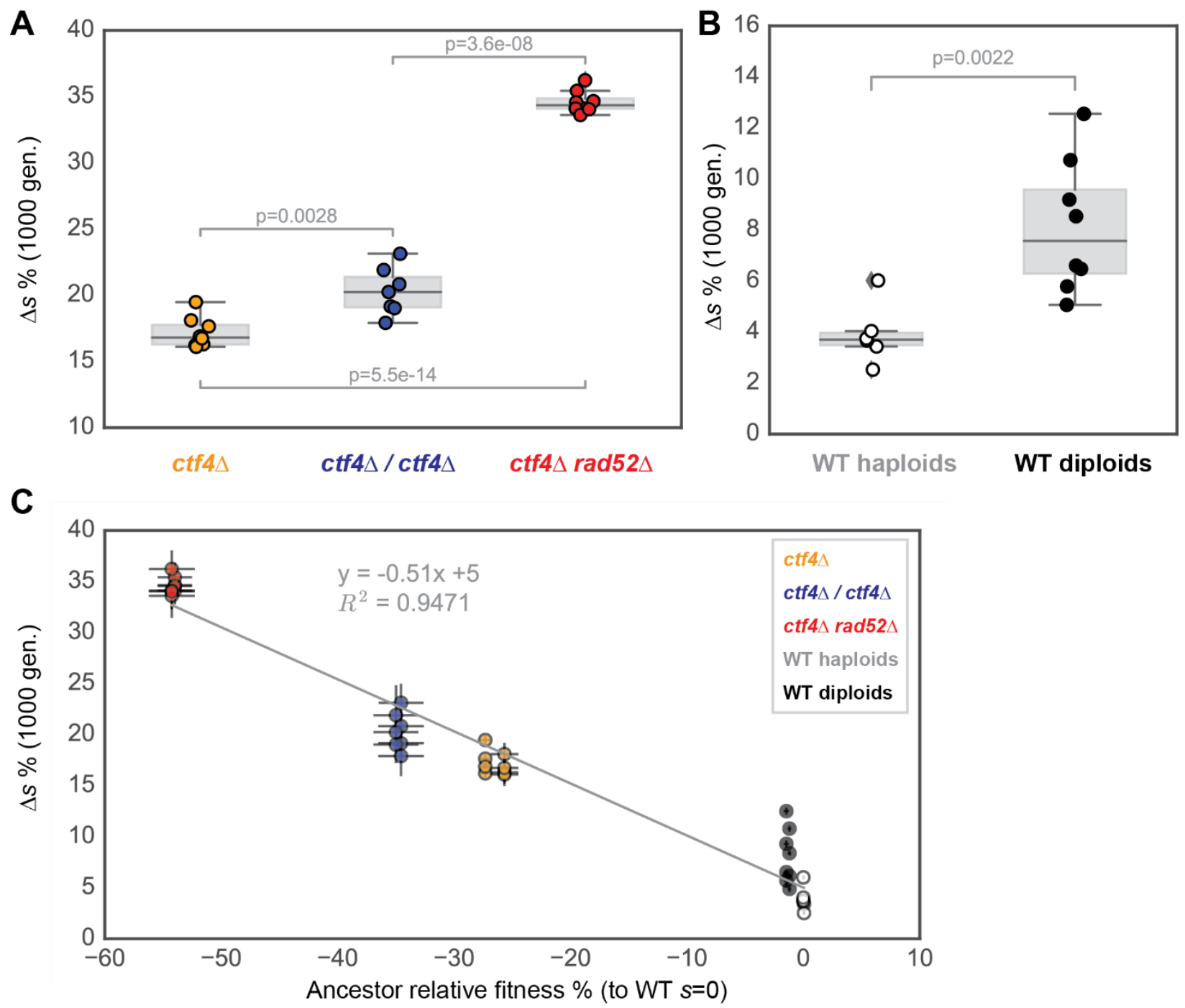

Ancestor relative fitness \% (to WT $s=0$ )

Figure 2: Fitness increase over 1000 generations. (A) The fitness increase over 1000 generations for strains experiencing constitutive DNA replication stress. $\Delta s$ is the difference between the fitness of the evolved populations and the fitness of their ancestors, with both fitnesses measured relative to that of cells that contain Ctf4 (CTF4) and have the same ploidy. (B). The fitness increase over 1000 generations for strains that contained wild-type CTF4. (C) shows the correlation between the fitness increase during evolution $(\Delta s)$ and the fitness defect of the ancestors relative to cells of the same architecture containing $\mathrm{Ctf} 4$ (the ancestor relative fitness). Fitness data of haploid strains (orange and white) is from [17]. Error bars represent standard deviations. Grey line represents linear regression between the datapoints. The P-values reported in figures are the result of $t$-tests assuming unequal variances (Welch's test). The data shown here are reported in or derived from Source data 1.

The majority of previous studies have suggested that diploids adapt more slowly than isogenic haploids $[15,69,70]$. Our diploid $c t f 4 \Delta / c t f 4 \Delta$ populations gained more fitness than $c t f 4 \Delta$ haploids over the course of 1000 generations (Figure 2A). Wild-type diploids increased more in absolute fitness than wild-type haploids (Figure $2 \mathrm{~B}$ ), suggesting that the discrepancy between our work 
and previous studies is not due to the presence of replication stress. Diploid populations recovered a slightly smaller fraction of their initial fitness defect compared to haploid and recombination-deficient strains (Figure 2-S2A). Collectively, our results suggest that ploidy is a poor predictor of the speed and extent of adaptation (Figure 2-S1). We conclude that the fitness of the ancestral cells is the major determinant influencing how much populations adapt, adding further support to the concept of 'declining adaptability'.

\section{Limited parallelism in adaptively mutated genes across genome architectures.}

We used whole-genome sequencing to understand the genetic basis of the evolutionary adaptation to DNA replication stress. For each different genome architecture, we sequenced all eight evolved populations, as well as four individual clones isolated from each of them. A total of 195, 276 and 905 genes or associated regulatory sequences were found mutated in evolved haploid, diploid, and recombination-deficient populations respectively (Figure 3A, Supplementary table 1). When normalized for DNA content, haploid (13 mutations/haploid genome) and diploid clones ( 9 mutations/haploid genome) had similar but statistically different mutation frequencies $\left(p=4 \times 10^{-6}\right.$, Welch's test, Figure 3-S1A) but recombination-deficient clones carried many more mutations, both in total (50/haploid genome, Figure 3-S1A) and as synonymous mutations (7/haploid genome, Figure 3-S1B). Our results agree with the reported increase in spontaneous mutagenesis in rad52 $\Delta$ cells [71-73].

We used statistical analysis to identify candidate adaptive mutations, by identifying genes that were mutated more often than expected by chance in independent populations (see Materials and methods for details). We performed this analysis both within individual genome architectures and across all three architectures. Using a false discovery rate of $5 \%$ we found a total 50 genes that met one or both criteria.

Only 8 of these genes were mutated in all three genome architectures (Figure 3A). This small number could be due to different adaptive strategies adopted by the different architectures, differences in the rate for the same mutation in different architectures, differences in the selective benefit the mutation conferred, or a combination of these three effects. We found 28 genes with putative adaptive mutations that are specific to individual genome architectures, as well as 14 genes with putative adaptive mutations in two architectures (Figure $3 \mathrm{~A}$, genes highlighted in boxes and Supplementary table 2). Analyzing gene ontology (GO) terms associated with the putative adaptive mutations revealed an enrichment of biological process terms associated with genome maintenance (Supplementary table 3). The genes that were significantly mutated in all genomic architectures included two that we had previously demonstrated to be adaptive in haploid ctf $4 \Delta$ cells: mutations in RAD9 inactivate the DNA damage checkpoint and those in SLD5 alter the function of the replicative helicase [17]. All three architectures also had mutations in RAD61 and PDS5, which are implicated in chromosome segregation [74,75], a cellular module shown to play a role during the adaptation of haploid $\operatorname{ctf} 4 \Delta$ populations [17]. 


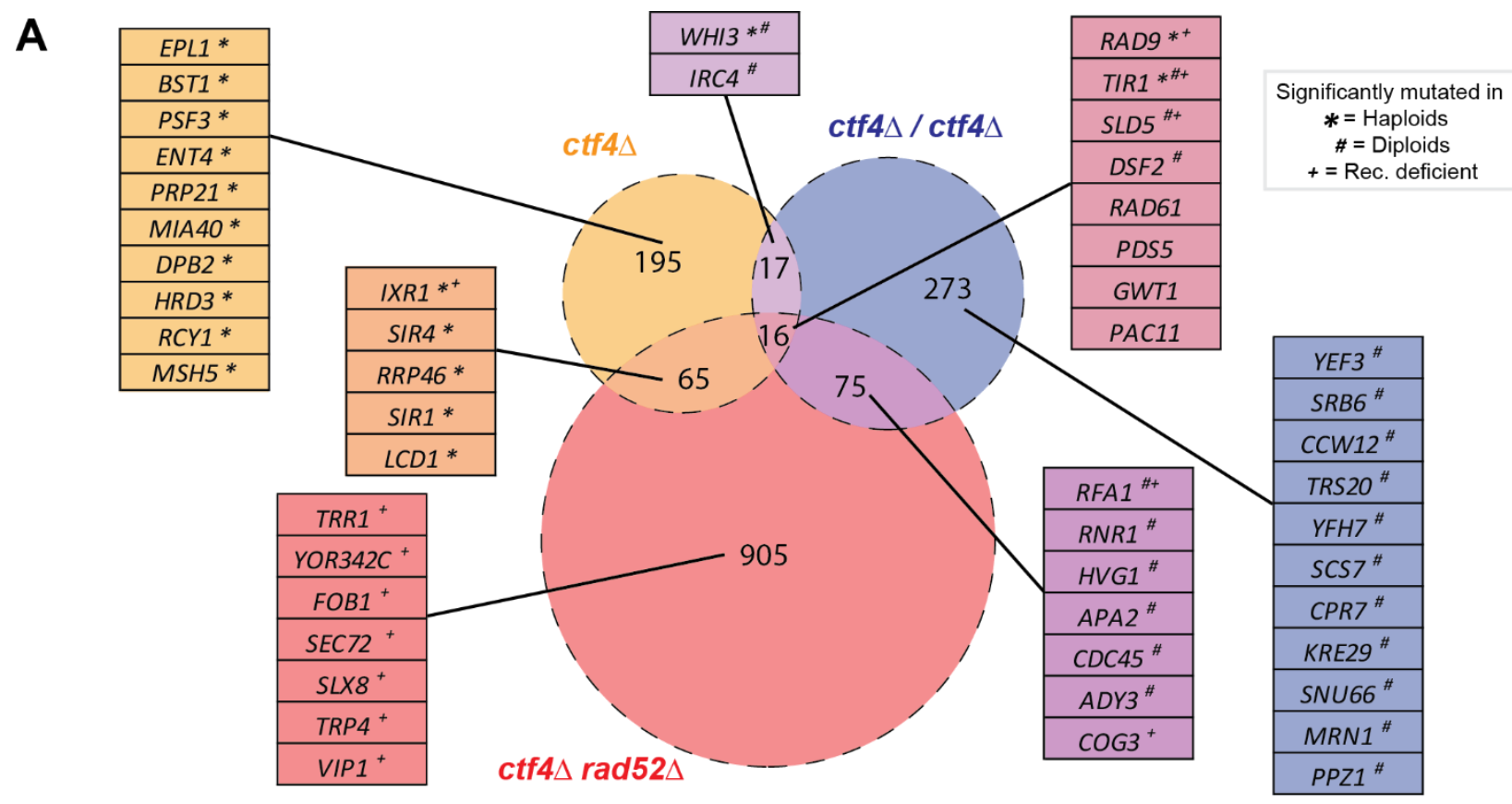

B

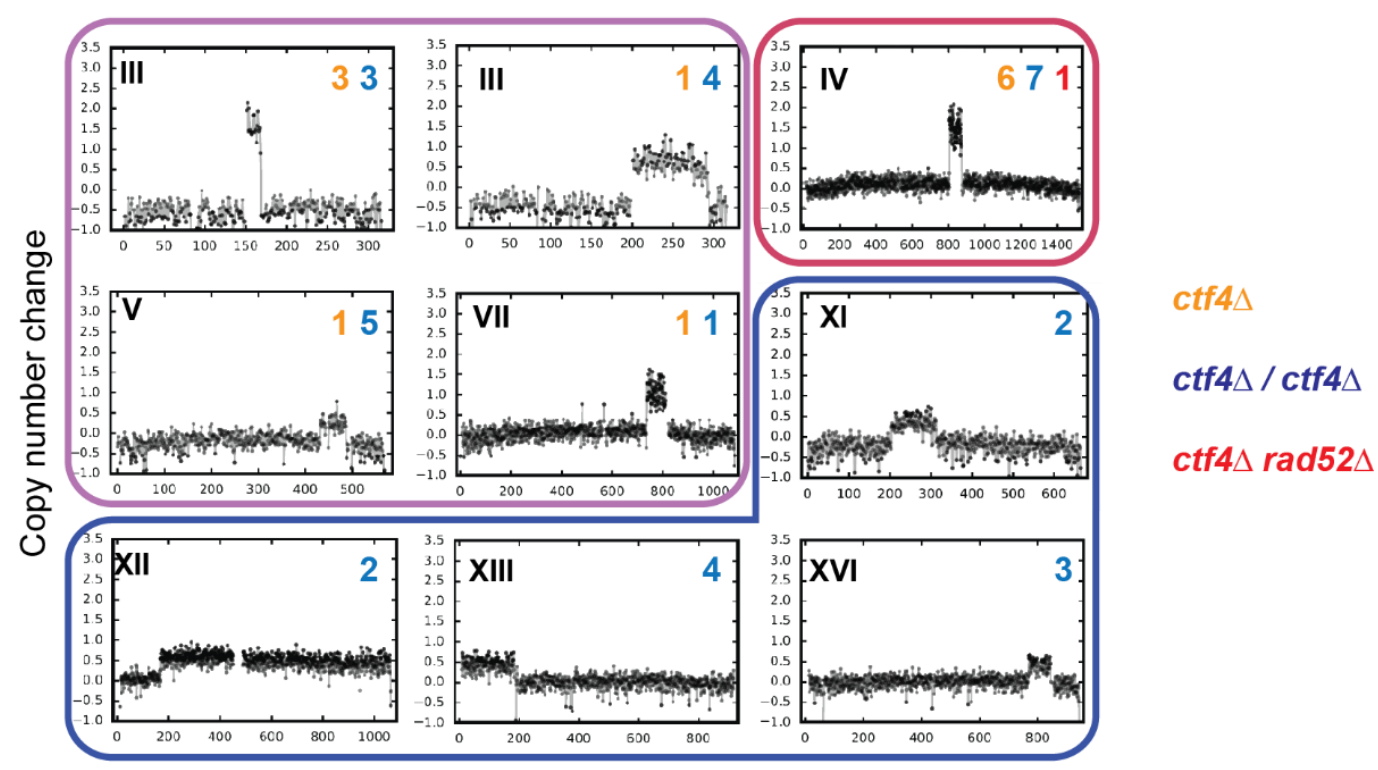

\section{Chromosome coordinates}

Figure 3: Putative adaptive mutations in the evolved clones. (A) Venn diagram representing the mutations (SNPs and small InDels 1-55bp) found in the evolved clones and populations. The area of the circles is proportional to the list of genes mutated at least once in the set of evolved clones for each genome architecture. The number within each sector represents number of genes which were mutated in one, two, or three genetic architectures. Tables include the genes whose mutations were significantly mutated in either global or architecture-specific analysis. Symbols next to the genes highlight that they were statistically significantly mutated in individual architectures ${ }^{*}$ refers to haploid, \# to diploid and + to recombination-deficient architectures, e.g. SLD5 ${ }^{+\#}$ means that the gene is found mutated in all three architectures, but is statistically significantly mutated only in diploid and recombinationdeficient populations). The identities of all the genes in the Venn diagram intersections are reported in Source data 3 (B) Copy number variations (CNVs) affecting different chromosomes (roman numbers) which appeared in at least two independent populations. Arabic numbers are the number of independent populations, in each genetic 
architecture (highlighted by a different color), in which the CNVs were detected. Copy number change refers to the fragment's gain or loss during the evolution experiment (i.e. +1 means that one copy was gained in haploid cells and that two copies were gained in diploid cells). Frames around the plots have the same color as the sectors in the Venn diagram, and encircle CNVs that have been found in one, two, or three genetic architectures. All the segmental amplifications detected are reported in Supplementary table 4.

224

We also examined the evolved genomes for changes in gene copy numbers (Copy Number Variations, CNVs) that might have played a role in adaptation. Many clones displayed an increased copy number of defined chromosome segments (segmental amplification) compared to the control populations (Supplementary table 4). Segmental amplifications were common in diploid clones (Figure 3-S1C). We inferred that segmental amplifications were putatively adaptive if the same chromosome region was found amplified in two clones isolated from independent populations (Figure 3B). Among these segmental amplifications, four CNVs were found only in evolved diploids, four were found in at least an evolved haploid and a diploid clone, and only one was detected in populations of all three genome architectures (Figure 3B). This latter fragment is located on chromosome IV and contains SCC2, which encodes a subunit of the cohesin loader complex. This essential complex is responsible for loading the proteinaceous cohesin ring that holds sister chromatids together until they segregate from each other during mitosis [76-78]. The gene encoding for the other subunit of the cohesin loader complex, SCC4, lies on the segment of chromosome $\mathrm{V}$ that was amplified in both haploid and diploid clones. We previously showed that extra copies of these genes alleviate the cohesion defects associated with the absence of Ctf4 in haploid cells and increase their fitness [17].

Overall, genome sequencing reveals that the identity of the genes carrying selected mutations varies depending on genome architecture. Nevertheless, we found evidence of common evolutionary strategies across different populations: Mutations affecting sister chromatid cohesion, DNA replication, and the DNA damage checkpoint, which were previously shown to drive adaptation to a form of DNA replication stress in haploid cells [17], are significantly mutated regardless of the genome architecture of the ancestral cells.

\section{Evolved diploids show adaptive loss of heterozygosity}

247 We analyzed the frequency of mutated reads in evolved diploid clones to estimate whether 248 mutations were homozygous or heterozygous (Figure 4-S1). We detected 36 homozygous 249 mutations across the evolved diploid clones (Figure 4A, Figure 4-S1B, Supplementary table 5). 250 Homozygous mutations were evenly distributed across the evolved clones analyzed and thus 251 were not the product of a single unrepresentative event (Figure 4-S1C). Individual clones often 252 carried multiple homozygous mutations on the same chromosome arm (Supplementary table 5) 253 suggesting that the loss-of-heterozygosity (LOH) was likely the consequence of mitotic 254 recombination events affecting large chromosomal segments [79]. In addition to homozygous 255 mutations, three genes were found to carry two different mutations, most likely one in each of 256 the two copies of the gene (double hits, Figure 4A, Supplementary table 5). Ten of the 257 homozygous or double hit mutations were abundant within their respective populations 
(mutation penetrance $>70 \%$, Figure 4B and 4-S2). Gene ontology analysis of the 39 genes with homozygous mutations or double hit alleles revealed the enrichment of many terms associated with genome maintenance (Supplementary table 6). One cluster of genes, linked by genetic and physical interactions, affected components of the replisome and factors involved in the DNA damage checkpoint (Figure 4B). Because these genes had been changed by two events (either two mutations, or a mutation followed by $\mathrm{LOH}$ ) and the altered forms had risen to high frequency in the evolved populations (Figure 4B), we argue that the inactivation or modification of many of these genes was selected for by constitutive DNA replication stress.

A

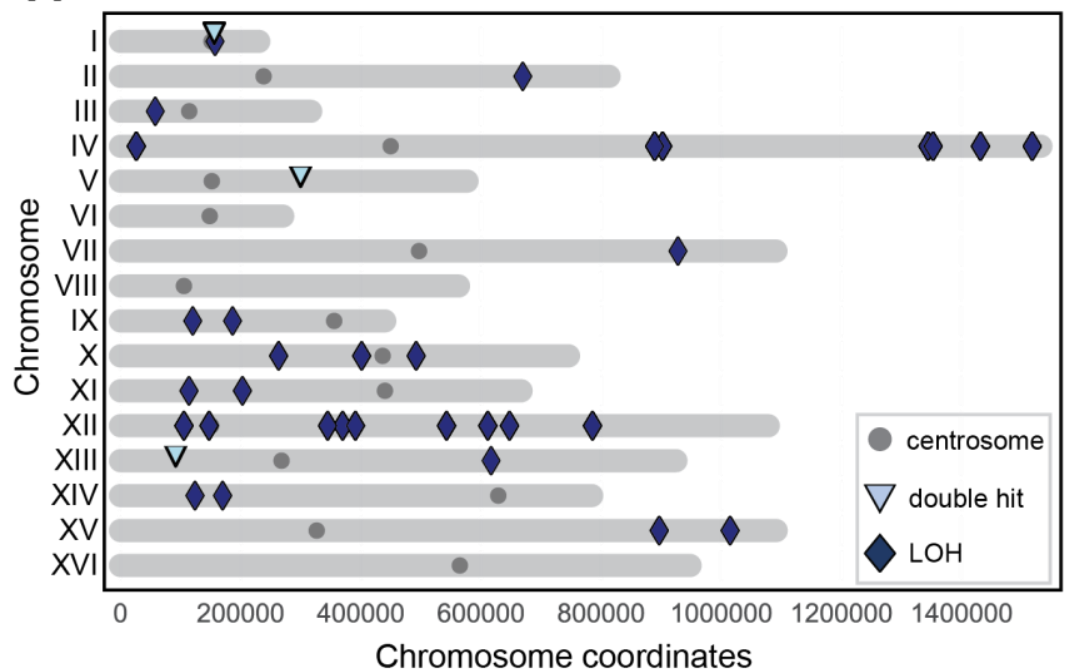

B

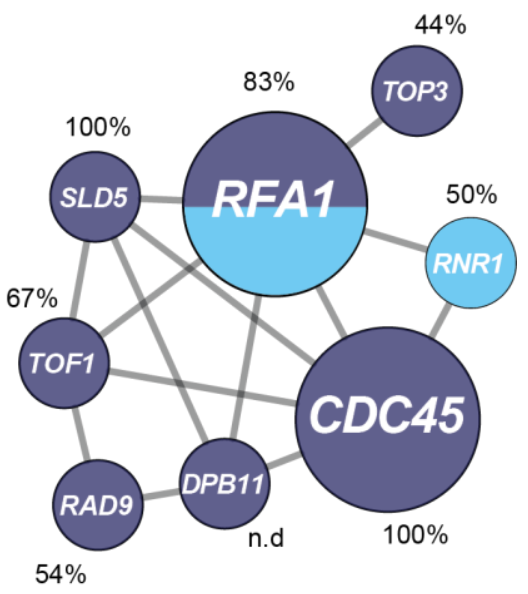

Figure 4: Homozygous mutations in diploids evolved in the presence of constitutive DNA replication stress. (A) Homozygous mutations (SNPs and small InDels of 1-55bp) detected in evolved ctf4 $\Delta /$ ctf $4 \Delta$ diploids clones distributed by chromosome (roman numbers). Dark blue diamonds represent loss of heterozygosity events (LOH). Light-blue triangles represent genes which contain two independent mutations (double hits) and the gray circles are the centromeres. The identities of the mutations shown here are reported in Supplementary table 5. (B) DNA replication and DNA damage checkpoint genes with mutations in both copies in evolved ctf4 $\Delta / \operatorname{ctf} 4 \Delta$ diploid clones. Gray lines represent evidence of genetic and physical interactions from the literature (https://string-db.org). Node diameter is proportional to the number of populations in which the gene was mutated. Dark blue represents $\mathrm{LOH}$ events, light blue represents double hits. Percentages refer to the average estimated frequency within populations of the clones carrying the respective mutation in homozygosity (LOH) or carrying both mutations (double hits).

\section{Genome architecture influences the distribution of adaptive mutants}

In addition to altering which genes get adaptive mutations, we hypothesize that genome architecture could affect evolutionary trajectories in two ways: changing the frequency at which different modules are altered and changing the distribution of the types of mutations that are selected (for example by altering the ratio of adaptive point mutations to copy number variation [73,80-84]). Our analysis revealed that all three architectures acquired putative adaptive mutations in three genome maintenance modules: sister chromatid cohesion, DNA replication and the DNA damage checkpoint. Out of the 23 populations analyzed, 18 of them contained at least one clone with mutations affecting all three of these modules, while all of them contain a 
clone with mutations in two of the three modules (Figure 5A). Despite the ubiquity of mutations in genome maintenance, the frequency of mutations in genes known to affect individual cellular modules varied between genome architectures: mutations affecting sister chromatid cohesion were underrepresented in recombination-deficient strains $\left(p=2.7 e-04, \chi^{2}\right.$ test) and there were fewer mutations affecting the DNA damage checkpoint in diploid clones $\left(p=0.03, \chi^{2}\right.$ test, Figure $6 A)$.

If amplifying the genes for the cohesin loader, SCC2 and SCC4, depended on homologous recombination between repeated sequences, the rate of amplification should be dramatically decreased in the recombination-deficient strain, which lacks Rad52. Analysis of the segmental amplifications containing SCC2 or SCC4 revealed that their boundaries correspond to three classes of repeated sequences: transposons (Ty elements), transposon-associated long terminal repeats (LTRs) and tRNA genes (Figure 5-S1 and Supplementary table 7). Recombination at these repeated elements has been previously shown to produce segmental amplifications selected under limited nutrient availability [85-87]. Amplification of SCC2 thus occurs as a result of homologous recombination between two different copies of the repeated sequences on chromosome IV, one on either side of SCC2 and amplification of SCC4 is due to similar events on chromosome $\mathrm{V}$. This dependence on homologous recombination explains the absence of segmental amplifications in all but one of the recombination-deficient clones (Figure $3 \mathrm{~B}$ and Supplementary table 4). Because changes affecting sister chromatid cohesion rely predominantly on gene amplifications (Figure 5C), we detect fewer mutations affecting this module in recombination-deficient strains (Figure $5 \mathrm{~A}$ ).

In haploid ctf4 $\triangle$ cells, inactivating the DNA damage checkpoint increases fitness [17]. The primary target for mutation is $R A D 9$, both because of its large size (3927 bp) and the presence of a run of 11 As, which repeatedly gain an extra nucleotide causing a frameshift mutation. Because this is a loss-of-function mutation [17], removing Rad9 function in diploids requires two events, thus reducing the probability of adapting by destroying the DNA damage checkpoint. Despite this difficulty, one diploid clone is homozygous for the frameshift mutation in RAD9, most likely as a result of a mutation in one copy of the gene followed by mitotic recombination or gene conversion that homozygosed this mutation and fully inactivated the checkpoint. The need to follow a two-hit pathway to fully inactivate the DNA damage checkpoint explains why fewer RAD9 mutations were found in diploids.

We conclude that although mutations affecting sister chromatid cohesion, DNA replication and DNA damage checkpoint are found in all the three genome architectures, the frequency at which we detect them depends on the genome architecture of an evolving population: gene amplification occurs much less frequently in recombination-deficient populations, making one pathway for improving sister chromatid cohesion less accessible, and recessive null mutations are rarer in diploid populations, blocking a major route by which haploids inactivate the DNA damage checkpoint. 
A \# gene mutations and amplifications

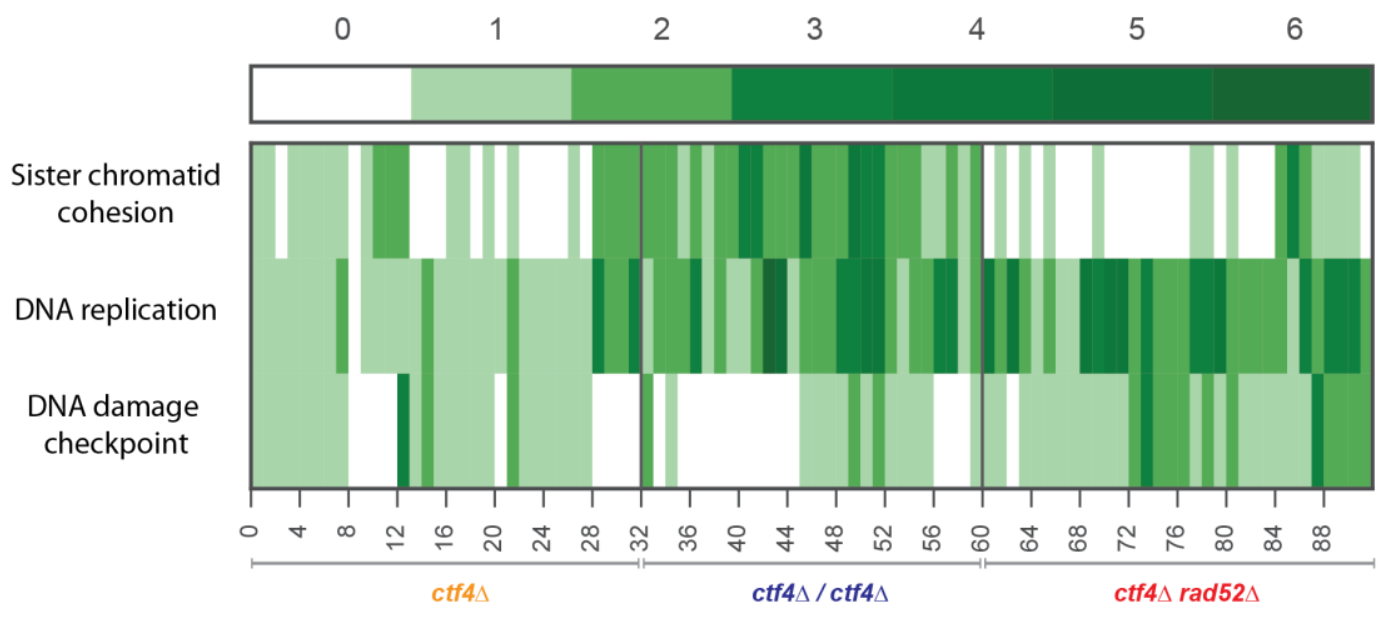

B

\# gene mutations

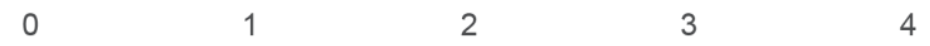

Sister chromatid cohesion

DNA replication

DNA damage checkpoint

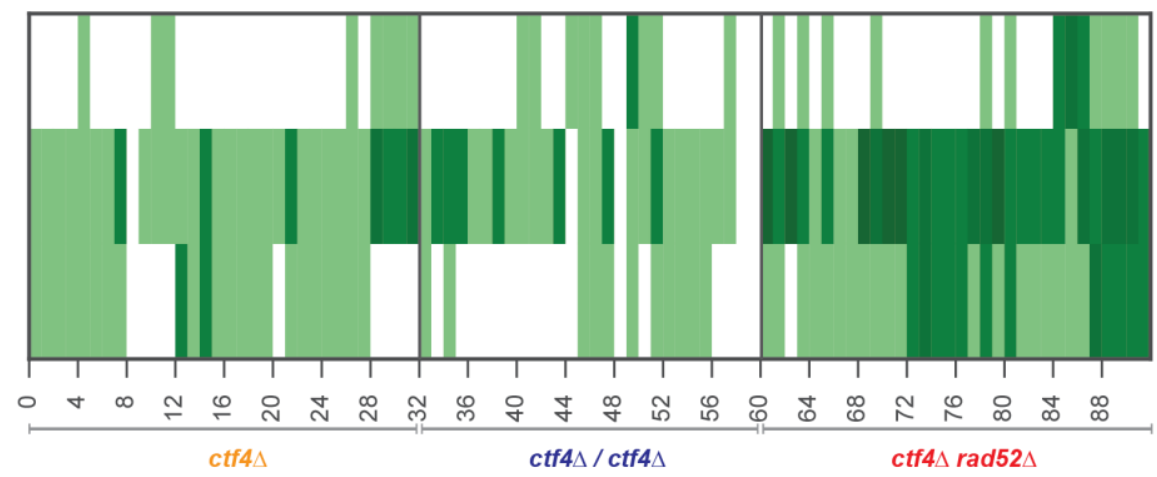

C
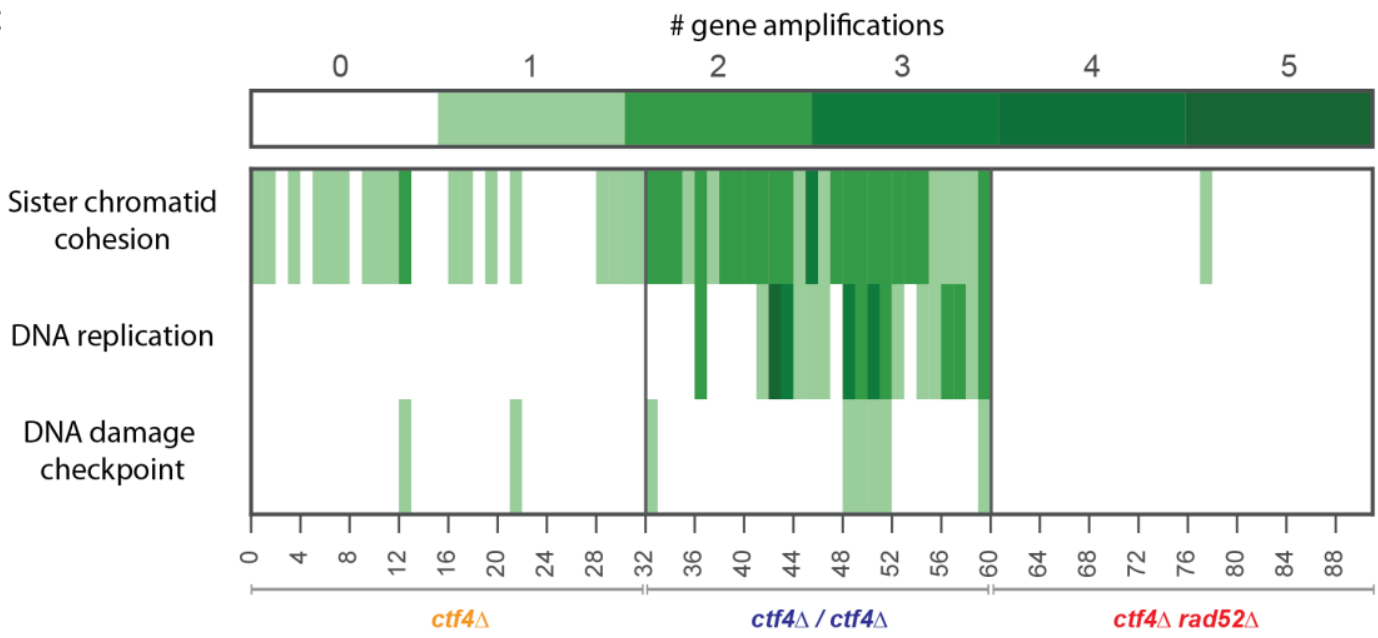

Figure 5: Mutations affecting sister chromatid cohesion, DNA replication and the DNA damage checkpoint. (A) Heatmaps representing all the mutations (SNPs, small InDels of 1-55bp and segmental amplifications) causally implicated in these phenotypes in the 92 sequenced evolved clones. (B) Frequency of SNPs and small InDels (1-55bp) affecting genes (Open reading frames and associated regulatory regions). (C) Frequency of genes associated with 
the three processes that are present on segmental amplifications. The number of hits and the identities of the genes which were mutated are reported in Source data 5.

\section{Mutations affecting sister chromatid cohesion, DNA replication and DNA damage checkpoint recapitulate evolution in all genome architectures.}

We asked whether genome architecture affected the benefit that mutations conferred as well as the frequency at which they were observed. To test this hypothesis, we chose a group of recurring mutations to validate their causality and measure their fitness benefit in different genome architectures. We mimicked the segmental amplification of chromosome IV (Figure 3B), affecting the cohesin loader SCC2, by integrating an extra copy of the gene at the URA3 locus (referred to as $2 x S C C 2$ ). Ixr1 is a transcription factor that indirectly affects DNA replication by positively regulating the production of deoxyribonucleotide triphosphates (dNTPs, [88]). The abundance of mutations causing premature stop codons in IXRI suggested that evolution selected for the inactivation of this gene (Supplementary table 8 ). We mimicked the loss of a functional Ixr1 protein by gene deletion (ixr1 1 ). Another recurrently mutated gene affecting DNA replication was SLD5, which encodes the subunit of the main replicative helicase (CMG, [89]) that is bound in vivo by Ctf4 [47]. SLD5 is an essential gene and our earlier analysis suggested that mutations like sld5-E130K reduced or altered the helicase's function [17]. Finally, the majority of mutations affecting the DNA damage checkpoint factor RAD9 resulted in early stop codons (Supplementary table 9), leading us to test the effect of the rad9 $\Delta$ gene deletion. These mutations were all previously demonstrated to be adaptive in haploid cells [17] and were used because they occurred, albeit at different frequencies, in all three (point mutations in SLD5 and RAD9 and amplification of SCC2) or at least two genome architectures (point mutations in IXR1 in both haploid populations).

We found that all four mutations were adaptive in all three genomic architectures. Remarkably, mutations affecting individual genes were adaptive even in architectures where they were never (IXR1 in diploids) or rarely detected (SLD5 in haploids, RAD9 in diploids and SCC2 in recombination-deficient strains). Three of the mutations we tested (SCC2 amplification, sld5E130K, and rad9D) conferred similar benefits in all three architectures, but the fourth, ixr $1 \Delta$, gave a much larger benefit in the recombination-deficient strain. One possibility is that the absence of Ixr1 reduces the frequency of double-stranded breaks associated with DNA replication in the absence of Ctf4. These breaks are lethal in the absence of Rad52 but can be repaired in the other two architectures.

For the diploid $c t f 4 \Delta / c t f 4 \Delta$ strain, we tested each mutation as a heterozygote (e.g. IXR1/ixr1 $\Delta$ ) and a homozygote (e.g. ixr1 $\Delta /$ ixr1 $1 \Delta$ ). The homozygous deletion of IXR1 gave a substantial fitness benefit (Figure 6A, dark blue), but strains heterozygous for the deletion were slightly less fit than their ancestors (Figure 6A, light blue). For the other three mutations (an extra copy of SCC2, sld5E130K, and rad9), the heterozygotes conferred a fitness benefit that was significant, albeit 
weaker than that of the homozygote. These results suggest that homozygous mutations can be selected by a two-step process in which both the first step, mutation in one copy of the gene, and the second, mutation in the other copy or loss of heterozygosity, confer a selective advantage. We conclude that, mutations affecting sister chromatid cohesion, DNA replication

371 and DNA damage checkpoint are beneficial in the presence of constitutive DNA replication stress

372 independently of the genome architecture of the strains.

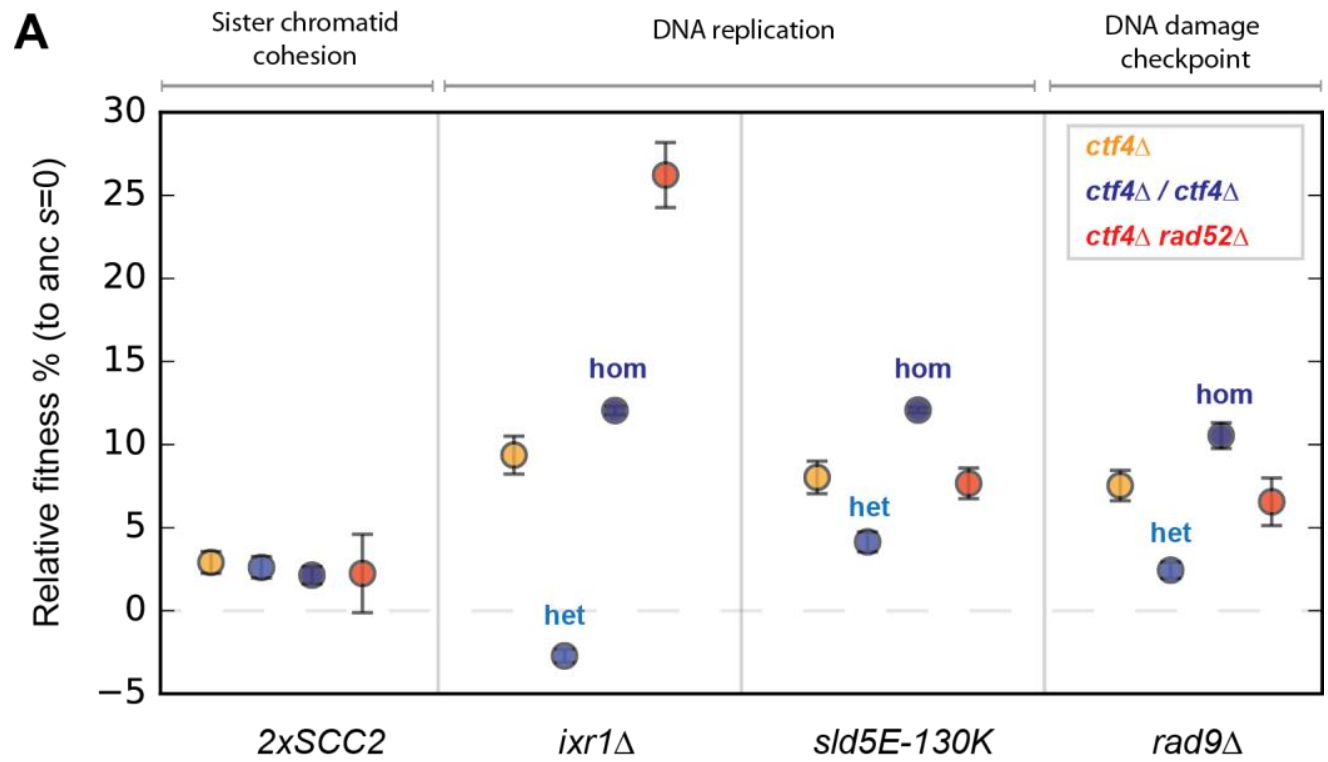

\section{B}

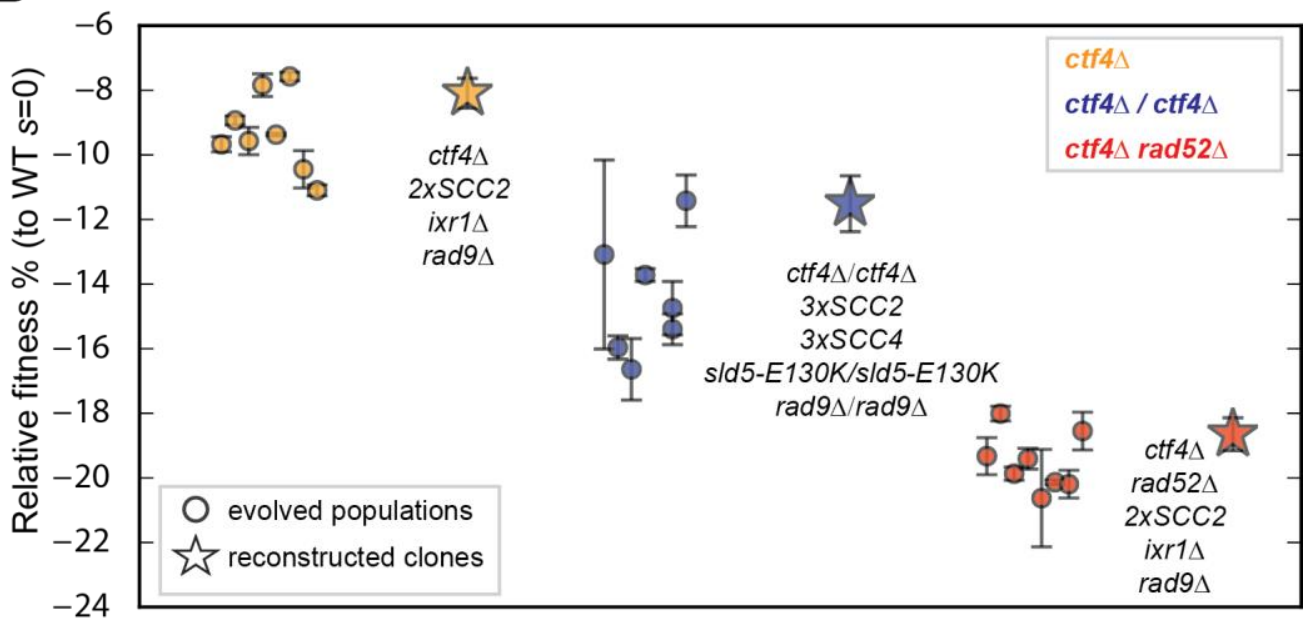

374 Figure 6: Reconstructed adaptive mutations can produce evolved fitness increases. (A) A subset of mutations affecting sister chromatid cohesion, DNA replication and DNA damage checkpoint were re-constructed in the ancestor cells for all three genomic architectures. The fitness of the re-constructed strains, relative to their respective $c t f 4 \Delta$ ancestor cells $(s=0)$ is depicted. For diploid strains, we replaced one (het) or two (hom) copies of the wild-type gene with the mutant allele. The fitness values shown here are reported in Source data 6a. (B) Reconstructed strains carrying combinations of adaptive mutations affecting sister chromatid cohesion, DNA replication and DNA damage checkpoint recapitulate the fitness of their respective evolved populations after 1000 generations. The most frequently mutated gene affecting DNA replication was chosen for the reconstruction in individual architectures (e.g. IXR1 was mutated more often that SLD5 in haploids whereas the reverse was true in 
diploids). Fitness data of haploid strains (orange) is from [17]. The P-values reported in figures are the result of ttests assuming unequal variances (Welch's test). The fitness values shown here are reported in Source data $6 \mathrm{~b}$.

Are mutations in sister chromatid cohesion, DNA replication and the DNA damage checkpoint enough to recapitulate the evolved fitness increase in all three genomic architectures? We previously showed that a haploid $c t f 4 \Delta$ strain carrying a mutation in all three of these modules recapitulated the fitness of the evolved strains (Figure 6B and [17]). We therefore asked if mutations in these three modules were sufficient to recapitulate 1000 generations of evolution in diploid and recombination-deficient strains. We constructed strains that carried mutations affecting sister chromatid cohesion, DNA replication and DNA damage checkpoint (Figure 6B), using mutations that had been found in the same genome architecture (Figure 3,4 and Supplementary table 1,4 ) and shown to be adaptive in isolation (Figure 6A).

For all three genome architectures, the reconstructed strains had a fitness that was similar to the evolved populations with the same architecture (Figure 6B). This result demonstrates that for all three genome architectures, acquiring mutations in all three modules (sister chromatid cohesion, DNA replication and DNA damage checkpoint) can recapitulate the fitness increase seen in response to evolution in the presence of DNA replication stress.

\section{DISCUSSION}

We previously showed that haploid cells adapt to a form of constitutive DNA replication stress by accumulating mutations affecting DNA replication, sister chromatid cohesion, and the DNA damage checkpoint [17]. We repeated this experiment in two other genomic architectures, a diploid and a recombination-deficient haploid strain, to ask which features of this evolutionary trajectory were general and which were specific to a particular genomic architecture. We find that the genes that acquire adaptive mutations, the frequency at which they are mutated, and the frequency at which these mutations are selected all differ between architectures but that mutations that confer strong benefits can occur in all three modules in each architecture. Engineering one mutation in each module into an ancestral strain lacking Ctf4 is enough to produce the evolved fitness increase in all three genomic architectures. Furthermore, reconstruction of a panel of mutations into all three architectures proved they are adaptive even in architectures where the affected genes were not found significantly mutated by the end of the experiment. Altogether our results demonstrate the existence of a common pathway for yeast cells to adapt to a form of constitutive DNA replication stress.

\section{The speed of adaptation to DNA replication stress depends on initial fitness not genome architecture}

What determines the rate of evolutionary adaptation? One possibility is genome architecture: beneficial, recessive mutations are harder to select in diploids [69,90] and gene amplification is less frequent in recombination-defective cells [91,92], suggesting that recombination proficient 
haploids should evolve faster than the other two genome architectures. Another explanation is that the initial fitness of the population is the principal factor in setting the rate of adaptation, with less fit ancestral populations increasing in fitness faster than more fit ones, a phenomenon referred to as declining adaptability [63]. We observed that adaptation rates scale linearly with the fitness difference between a mutant ancestor and a non-mutant wild-type, showing that the recovery from DNA replication stress follows the declining adaptability documented in other experimental conditions.

Previous work has produced a variety of results with the majority reporting that increasing ploidy decreases the rate of evolution $[15,69,70]$ and a minority reaching the opposite conclusion $[14,16]$. We suggest that this variability arises because genome architecture affects two aspects of the mutations that produce adaptation: the frequency at which they occur and the selective advantage they confer. Thus, we find that defects in recombination reduce the rate of gene amplification and diploidy makes the accumulation of fully recessive mutations require two successive genetic events rather than a single mutation. Despite these barriers, our diploids and recombination-defective haploids evolved faster than recombination-proficient haploids. We can only speculate on why this is so but note that the recombination-proficient strains have a higher mutation rate and diploids may be more resistant to the unbalanced gene dosage caused by segmental amplifications. Our results thus go against the trend of slower adaptation in diploids as compared to haploids reported by the majority other studies $[15,69,70]$. This effect is not limited to populations experiencing DNA replication stress (Figure 2A) but is also present in control wild-type populations (Figure 2B). Our results support the idea that the details of genotypes, selections, and experimental protocols can determine the effect of ploidy on adaptation.

We engineered the same mutations into all three genome architectures to ask if the benefit of individual mutations depended on either genome architecture or the fitness of the recipient strain. For three of the four mutations we tested (sld5-E130K, rad94, and 2xSCC2) their effect was independent of both these variables. The fourth (ixr1 $\Delta$ ) showed diminishing return epistasis, defined as an adaptive mutation producing a smaller benefit when present in fitter genetic backgrounds [65]. This effect has been invoked to explain declining adaptability and is thought to arise from the complex pattern of epistasis between the adaptive mutations and the set of alleles present in the genetic background [68,93-96]. Our results therefore agree with previous reports observing declining adaptability across strains with different initial fitness but largely fail to observe diminishing return epistasis as a potential justification of this phenomenon. Our experiments and two previous evolutionary repair experiments (Hsieh et al., 2020; Laan et al., 2015) both show interactions that are approximately additive between different selected mutations. The reasons for this difference are currently unknown.

\section{General principles of adaptation to constitutive DNA replication stress}

Evolutionary change can be examined at multiple levels from the details of adaptive mutations in individual genes, the identity of the functional modules in which the mutations lie, cellular 
phenotypes, such as the integrity of the DNA damage checkpoint, to overall reproductive fitness. For each of these levels, we examined the effect of genome architecture on the adaptation to a form of constitutive DNA replication stress. At both ends of this scale we see substantial variation: genome architecture influences the frequency at which mutations occur, the fitness benefit they confer, and the extent of overall adaptation. In the middle, we see more conservation, with all three architectures accumulating mutations in the same three functional modules, suggesting that they all follow similar phenotypic paths by increasing the stability of the linkage between sister chromatids, stabilizing replication forks, and inactivating the DNA damage checkpoint. To test this hypothesis, we reconstructed mutations from all three modules into the ancestral, Ctf4deficient strains of all three genome architectures. This manipulation reproduced fitness observed after 1000 generations of evolution leading us to conclude that the principles that govern evolutionary adaptation to constitutive DNA replication stress are conserved across the genome architectures we examined.

\section{Molecular mechanisms affecting adaptation in diploid cells.}

The phenotype of heterozygous mutations in diploid cells depends on their dominance. Although loss-of-function mutations are often assumed to be fully recessive, a substantial minority have detectable phenotypes as heterozygotes (referred to as semi-dominance or haploinsufficiency), typically much weaker than that of the homozygous loss-of-function mutations [97]. The phenotype of the heterozygote determines whether mutations will be selected for in diploid populations [98,99]. As an example, loss-of-function mutations in IXR1 were repeatedly found in haploid and recombination-deficient populations but were not present in evolved diploid strains. This observation is consistent with the slight fitness cost of the heterozygous IXR1 deletion, despite the strong benefit provided by the homozygous deletion: the cost of the heterozygote acts as a barrier that prevents access to the beneficial homozygous mutations. Like IXR1, deletion of both copies of RAD9 has a strong fitness benefit in diploids, but in this case there is a small fitness benefit in the rad9 $\triangle / R A D 9$ heterozygotes providing a path to inactivating the DNA damage checkpoint in which both steps increase fitness. This two-step path in diploids is less favorable than the one-step path that inactivates the checkpoint in haploids and likely accounts for the lower frequency of mutations affecting RAD9 and other known checkpoint genes in evolved diploid clones (Figure 5 and Source data 5).

Two-step paths affected the replication machinery or DNA damage checkpoint in 9 of the 28 diploid clones; 2 involved independent mutations in the same gene and 8 produced homozygous mutations due to gene conversion or mitotic recombination (Figure 4, one clone contained two homozygous mutations). The high frequency of these events suggests that these mutations conferred some advantage even as heterozygotes. For one mutation, sld5-E130K, we verified the fitness benefit of the s/d5-E130K/SLD5 heterozygote, which suggests that the mutant subunit is present in a fraction of the genome-wide replicative helicases, where it helps to stabilize replication forks in the absence of $\mathrm{Ctf} 4$. Mitotic recombination can then homozygose the mutation, eliminating the wild-type Sld5 protein and stabilizing all replisomes. The genome 
architecture of diploid cells thus explains both the reduced frequency of mutations in RAD9 and dictates which mutations affecting DNA replication are ultimately selected (e.g. IXRI versus $S L D 5)$. We suggest that semi-dominant mutations are an important intermediate in the selection of loss-of-heterozygosity during the evolution of diploid, somatic cells.

\section{Molecular mechanisms affecting adaptation in recombination-deficient cells.}

Recombination-deficient clones have much fewer segmental amplifications affecting the genes for the cohesin loader, SCC2 and SCC4 (Figure 5C). The lack of the recombinase protein Rad52 in recombination-deficient strains dramatically reduces their ability to achieve segmental amplifications. Only one event, likely mediated by Rad52-independent recombination [100-103], was found in the recombination-deficient evolved clones (Figure 3B and Supplementary table 4). The other mutations in these strains that are likely to affect sister chromatid cohesion were point mutations affecting other proteins involved in pairing and segregation of chromosomes such as Pds5 and Rad61 (Figure 5B). The higher number of point mutations in this and other modules is likely the consequence of increased spontaneous mutagenesis imposed by the lack of homologymediated repair of spontaneously occurring DNA lesions (Figure 3-S1,2, [104]). The genome architecture of recombination-deficient strains thus explains both the reduced frequency of gene amplification affecting sister chromatid cohesion and the increased number of point mutations detected in evolved clones (Figure 6B).

\section{Impact of genome architecture on evolutionary adaptation to DNA replication stress}

Our analysis argues that although genome architecture doesn't affect the ability of cells to adapt to DNA replication stress or the modules that alter to produce adaptation, it does affect the mutational pathways by which different architectures adapt.

If the major adaptive mechanisms are shared, how do we explain the limited number of genes that are mutated in all conditions and the genes that are uniquely and significantly mutated in specific genome architectures (Figure $3 \mathrm{~A}$ )? There are several hypotheses: some of these genes may be false positives, mutations in these genes confer benefits in more than one architecture but were only detected in a single architecture, and mutations in these genes are substantially more, or exclusively, beneficial in one architecture. The fact that our reconstructed strains recapitulate the fitness of the evolved populations with a minimal set of changes in genome maintenance suggests that architecture-specific mutations play a marginal role in adaptation. Of the 22 genes that were mutated in at least two architectures, 10 have known roles in DNA replication, repair, or sister chromatid cohesion, whereas only 5 of the 28 that were uniquely mutated in one architecture have such a role $\left(p=0.034, \chi^{2}\right.$ test). The fitness of the reconstructed strains (Figure 6A,B) also implies that the most important mutations for the adaptation to DNA replication stress are those that were found in multiple architectures (Figure 3A). Mutations that were never (ixr1), or rarely ( $2 x S C C 2$ and rad9) seen in a given genome architecture, nevertheless increased fitness in all three architectures. This finding suggests that caution should be used in inferring adaptive strategies from lists of genes that have been mutated more often than 
expected by chance, especially when comparing populations that were evolved under different conditions or have different ancestral genotypes. We argue that experimental dissection of adaptive trajectories is needed to distinguish the mutations that make major contributions to adaptation from minor players and mutational noise.

The distribution of mutations with known phenotypic effects suggests that genome architecture controls how much adaptation in different functional modules contributes to the overall response to selective pressure. Mutations that break the DNA damage checkpoint by inactivating $R A D 9$ are less frequent in diploid populations than they are in haploids, and amplifications of cohesin loader genes are less frequent in recombination-deficient populations than they are in recombination-proficient ones. Although we suspect that some of the diploid populations have found alternate genetic routes to partially or fully inactivate the DNA damage checkpoint and that some of the recombination-defective populations have selected other mutations that improve sister chromatid cohesion, these changes are likely to be less effective than those that were frequent in other genome architectures.

\section{Implications for cancer and natural evolution}

We previously speculated that evolutionary processes similar to the adaptation to constitutive DNA replication stress could assist the evolution of cancer cells and organisms in nature [17]. Tumor cells often experience DNA replication stress as a consequence of oncogene activation, promoting genetic instability and increasing the rate of cell death $[19,22]$. While generated through a different mechanism (unrestrained proliferation, rather than replisome perturbation), oncogene induced DNA replication stress produces cellular consequences [25] which are remarkably similar to the one described in the absence of $\mathrm{Ctf4}$, such as the accumulation of ssDNA gaps, stalled and reversed forks $[17,48,49]$, genetic instability $[49,51,52]$ and DNA damage response activation $[47,50]$. Based on these similarities we speculate that the evolutionary adaptation to DNA replication stress could reduce its negative effects on cellular fitness and thus assist tumor evolution. In natural populations, interference with DNA replication, through the fixation of deleterious mutations, perturbation by selfish genetic elements or toxins secreted by antagonistic organisms could generate similar evolutionary adaptation. The consequences of these evolutionary processes could rewire genome maintenance modules to alter cancer cells' physiology and generate molecular diversity in nature [105]. For these scenarios to be plausible, the evolutionary adaptation to DNA replication stress needs to happen over short timescales. In cancer, it would need to happen over the limited somatic evolution that accompanies cancer progression. In nature, it would need to occur before the perturbed organisms are outcompeted by unaffected ones or block the source of DNA replication stress. It could be argued that some genome architectures, such as diploidy or reduced recombination, could reduce the likelihood of evolving new strategies for genome replication and maintenance. In our hands, however, these genome architectures do not preclude rapid, evolutionary adaptation to constitutive DNA replication stress: loss of heterozygosity or increased mutation rates, which have both been documented in cancer $[106,107]$ and natural evolution $[108,109]$, allow cells to circumvent these 
obstacles and adapt quickly to a severe perturbation of the replication machinery. One rapidly evolving clade of yeasts has lost RAD9 [110], the primary target of checkpoint-inactivating mutations in our experiments. Similarly, many cancers inactivate the DNA damage response during tumor evolution [111,112]. We suggest that the careful examination of the genomic sequences of tumors and fast-evolving species might reveal signs of adaptation that mirror the molecular evolution we have described.

\section{MATERIALS AND METHODS}

\section{Strains}

All strains were derivatives of a modified version (Rad5 ${ }^{+}$) of S. cerevisiae strain W303 (leu2-3,112 trp1-1 can1-100 ura3-1 ade2-1 his3-11,15, RAD5). Supplementary table 10 lists each strain's genotype. The ancestors of wild-type, ctf4 $\Delta$ and $\operatorname{ctf} 4 \Delta$ rad52 $\Delta$ strains were obtained by sporulating a CTF4/ctf4A RAD52/rad52 $\Delta$ heterozygous diploid. This was done to minimize the selection acting on the ancestor strains before the beginning of the experiment. CTF4/CTF4 and ctf $4 \Delta /$ ctf $4 \Delta$ diploid strains were obtaining by mating freshly generated MATa and MAT $\alpha$ haploid strains.

\section{Media and growth conditions}

Standard rich medium, YPD (1\% Yeast-Extract, 2\% Peptone, 2\% D-Glucose) was used for the evolution experiment and all fitness measurements.

\section{Experimental evolution}

All populations used in the evolution experiment (EVO1-40) were initially inoculated in glass tubes containing $10 \mathrm{ml}$ of YPD with yeast colonies of the relative genotype. Glass tubes were placed in roller drums at $30^{\circ} \mathrm{C}$ and grown for $24 \mathrm{hr}$. Daily passages were done by diluting $10 \mu \mathrm{l}$ of the previous culture into $10 \mathrm{ml}$ of fresh YPD (1:1000 dilution, allowing for approximately 10 generations/cycle). All populations were passaged for a total of 100 cycles ( $\approx 1000$ generations). In this regime, the effective population size is calculated as $N_{0} \times g$ where $N_{0}$ is the size of the population bottleneck at transfer and $g$ is the number of generations achieved during a batch growth cycle and corresponds to approximately to $10^{7}$ cells. After 1000 generations four evolved clones were isolated from the populations evolved in the absence of Ctf4 (EVO1-24) by streaking cells on a YPD plate (total of 96 clones isolated). To capture the within-population genetic variability we selected the clones displaying the largest divergence of phenotypes in terms of resistance to genotoxic agents (methyl-methanesulfonate, hydroxyurea and camptothecin). All final populations and clones isolated from them were mixed with $800 \mathrm{ml}$ of $30 \% \mathrm{v} / \mathrm{v}$ glycerol and stored at $-70^{\circ} \mathrm{C}$ for future analysis. The evolution of haploid, diploid, and recombination defective strains, all lacking CTF4, and wild-type haploid and diploid strains were all carried out in parallel.

\section{Whole genome sequencing}


610 Genomic DNA library preparation was performed with an Illumina (RRID:SCR_010233, San Diego, 611 CA, US) Nextera DNA Library Prep Kit. Libraries were then pooled and sequenced either with an 612 Illumina HiSeq 2500 (125bp paired end reads) or an Illumina NovaSeq (150 bp paired end reads).

613 The SAMtools software package (RRID:SCR_002105, samtools.source-forge.net) was then used 614 to sort and index the mapped reads into a BAM file. GATK (RRID:SCR_ 001876, 615 www.broadinstitute.org/gatk; McKenna et al., 2010) was used to realign local indels, and VarScan 616 (RRID:SCR_006849, varscan.sourceforge.net) was used to call variants. Clones and populations 617 were sequenced at approximately the following depths: 25-30X for haploid clones, 50-60X for 618 diploid clones, 50-60X for haploid populations and 120-130X for diploid populations. Mutations 619 were found using a custom pipeline written in Python (RRID:SCR_008394, www.python.org). The 620 pipeline (github.com/koschwanez/mutantanalysis) compares variants between the reference 621 strain, the ancestor strain, and the evolved strains. Variants found in less than $25 \%$ and $35 \%$ of 622 the reads in haploid and diploid populations respectively were discarded, since many of these 623 corresponded to misalignment of repeated regions. For clone sequencing, only variants found in 624 more than $75 \%$ of the reads in haploids and $35 \%$ of the reads in diploids (to account for 625 heterozygosity) were considered mutations. The frequency of the reads associated with all the variants detected are reported in Supplementary table 1. A variant that occurs between the ancestor and an evolved strain is labeled as a mutation if it either (1) causes a substitution in a coding sequence or (2) occurs in a regulatory region, defined as the $500 \mathrm{bp}$ upstream and downstream of the coding sequence. Gapped alignments of the 150 paired-end reads in our data set permit the identification of small indels ranging in size from 1-55 bp using VarScan pileup2indel tool [113]. All small indels (and the respective sequence affected) are listed together with SNPs in Supplementary table 1.

\section{Identification of putative adaptive mutations}

634 To identify genes and cellular modules targeted by selection we used the following methods:

635 Parallel evolution of genes: This method relies on the assumption that those genes that have 636 been mutated significantly more than expected by chance alone, represent cases of parallel evolution among independent lines. The mutations affecting those genes are therefore considered putatively adaptive. The same procedure was used independently on the mutations

640 We first calculated per-base mutation rates as the total number of mutations in coding regions 641 occurring in a given background, divided by the size of the coding yeast genome in bp (including $6421000 \mathrm{bp}$ per ORF to account for regulatory regions)

$$
\lambda=\frac{S N P s+\text { indels }}{b p \text { coding }}
$$

If the mutations were distributed randomly in the genome at a rate $\lambda$, the probability of finding $\mathrm{n}$ mutations in a given gene of length $\mathrm{N}$ is given by the Poisson distribution: 
646

647

648

649

650

651

652

653

654

655

656

657

658

659

660

661

662

663

664

665

666

667

668

669

670

671

672

673

674

675

676

677

678

679

680

681

$\mathrm{P}(n$ mutations $\mid$ gene of length $N)=\frac{(\lambda N)^{n} e^{-\lambda N}}{n !}$

For each gene of length $\mathrm{N}$, we then calculated the probability of finding $\geq \mathrm{n}$ mutations if these were occurring randomly.

$$
P(\neq \geq n \text { mutations } \mid \text { gene of length } N)=\sum_{k=n}^{\infty} \frac{(\lambda N)^{n} e^{-\lambda N}}{k !}=1-\frac{\Gamma(n+1, \lambda N)}{n !}
$$

(Where $\Gamma$ is the upper incomplete gamma function) which gives us the $p$-value for the comparison of the observed mutations with the null, Poisson model. In order to decrease the number of false positives, we then performed multiple-comparison corrections. The more stringent Bonferroni correction ( $\alpha=0.05$ ) was applied on the wild-type evolved mutations dataset, while BenjaminiHochberg correction ( $\alpha=0.05$ ) was used for the ctf $4 \Delta$ mutation dataset. Genes that were found significantly selected in the evolved wild-type populations (after Bonferroni correction) were removed from the list of evolved ctf $4 \Delta$ strains (genes mutated in wild-type evolved populations were removed from the $\operatorname{ctf} 4 \Delta$ and $\operatorname{ctf} 4 \Delta$ rad52 $\Delta$ datasets and genes mutated in wild-type diploid populations were removed from the $\operatorname{ctf} 4 \Delta$ /ctf $4 \Delta$ dataset). We argue that since these genes were targets of selection even in wild-type cells, they are likely involved in processes that are unrelated to DNA replication and are instead associated with adaptation to sustained growth by serial dilutions. Supplementary table 2 lists the mutations detected in evolved clones and populations, after filtering out those that occurred in genes that were significantly mutated in the respective wild-type populations. Genes significantly selected are shown in dark grey (after BenjaminiHochberg correction with $\alpha=0.05)$.

Parallel evolution of cellular modules: Lists of genes that had been mutated more frequently than expected by chance (Supplementary table 2) or found homozygously mutated in diploid strains (Supplementary table 5) were further analyzed for gene ontology (GO) terms enrichment. Lists of mutations were input as 'multiple proteins' in the STRING database, which reports on the network of interactions between the input genes (https://string-db.org). The 'biological process' terms enriched in the significantly or homozygously mutated genes are listed in Supplementary tables 3 and 6 respectively.

\section{Gene ontology (GO) enrichment analysis}

The list of genes with putatively selected mutations (Figure 3A) or homozygous mutations in diploids (Figure 4) were input as 'multiple proteins' in the STRING database, which reports on the network of interactions between the input genes (https://string-db.org). The GO term enrichment analysis provided by STRING are reported in Supplementary Table 3 and Supplementary Table 6 respectively. Briefly, the strength of the enrichment is calculated as $\log _{10}(\mathrm{O} / \mathrm{E})$, where $\mathrm{O}$ is the number of 'observed' genes in the provided list (of length $\mathrm{N}$ ) which belong to the GO-term, and $\mathrm{E}$ is the number of 'expected' genes we would expect to find matching the GO-term providing a list of the same length $\mathrm{N}$ made of randomly picked genes. $\mathrm{P}$ values are computed using a Hypergeometric test and corrected for multiple testing using the 
682 Benjamini-Hochberg procedure. The resulting P-values are represented as 'False discovery rate' 683 in the supplementary tables and describe the significance of the GO terms enrichment [114]."

684

685

686

687

688

689

690

691

692

693

694

695

696

697

698

699

700

701

702

703

704

705

706

707

708

709

710

711

712

713

714

715

716

717

718

\section{Fitness assays}

To measure relative fitness, we competed the ancestors and evolved strains against reference strains. Cells were competed against reference strains with the same ploidy. Wild-type refence strains were used for the competition assays in all the figures, except for those in Figure 6A where ctf4A reference strains were used instead. A pFA6a-prACT1-yCerulean-HphMX4 plasmid was digested with Agel and integrated at one of the ACT1 loci of the original heterozygous diploid (CTF4/ctf4A RAD52/rad52A) strain. This allows for the expression of fluorescent protein yCerulean under the strong actin promoter. The heterozygous diploid was then sporulated and dissected to obtain fluorescent wild-type, ctf4 $\Delta$ or ctf4 $\Delta$ rad52 $\Delta$ reference haploid strains. ctf $4 \Delta$ /ctf4 $\Delta$ diploid reference strains were obtained by mating two ctf4 $\Delta$ haploid strains, one of which expressed yCerulean under the ACT1 promoter. For measuring a strain's relative fitness, $10 \mathrm{ml}$ of YPD were inoculated in individual glass tubes with either the frozen reference or test strains. After $24 \mathrm{hrs}$. the strains were mixed in fresh $10 \mathrm{ml}$ YPD tubes at a ratio dependent on the expected fitness of the test strain compared to the reference (i.e. 1:1 if believed to be nearly equally fit) and allowed to proliferate at $30^{\circ} \mathrm{C}$ for $24 \mathrm{hrs} .10 \mu \mathrm{l}$ samples were taken from this mixed culture (day 0 ) and the ratio of the two starting strains was immediately measured. Tubes were then cultured following in the same conditions as the evolution experiment by diluting them 1:1000 into fresh medium every $24 \mathrm{hrs}$ for 2-4 days, monitoring the strain ratio at every passage. Samples were analyzed by flow cytometry (Fortessa, BD Bioscience, RRID:SCR_013311, Franklin Lakes, NJ, US). Strain ratios and the number of generations occurred between samples were calculated using a custom Python script utilizing the FlowCytometryTools package (https://pypi.org/project/FlowCytometryTools/). Ratios, $r$, were calculated based on the number of fluorescent and non-fluorescent events detected by the flow cytometer:

$$
r=\frac{\text { NonFluorescent }_{\text {events }}}{\text { Fluorescent }_{\text {events }}}
$$

Generations between time points, $g$, were calculated based on total events measured at time 0 hrs and time $24 \mathrm{hrs}$ :

$$
g=\frac{\log _{10}\left(\text { Events }_{t 24} / \text { event }_{t 0}\right)}{\log _{10} 2}
$$

Linear regression was performed between the $\left(\mathrm{g}, \log _{e} r\right)$ points for each sample. Relative fitness was calculated as the slope of the resulting line. The mean relative fitness, $s$, was calculated from measurements obtained from at least three independent biological replicates. Error bars represent standard deviations. The P-values reported in figures are the result of t-tests assuming unequal variances (Welch's test).

\section{Copy number variations (CNVs) detection by sequencing}

Whole genome sequencing and read mapping was done as previously described. The read-depths for every unique $100 \mathrm{bp}$ region in the genome were then obtained by using the VarScan 
copynumber tool. A custom pipeline written in python was used to visualize the genome-wide CNVs. First, the read-depths of individual $100 \mathrm{bp}$ windows were normalized to the genome-wide median read-depth to control for differences in sequencing depths between samples. The coverage of the ancestor strains was then subtracted from the one of the evolved lines to reduce the noise in read depth visualization due to the repeated sequences across the genome. The resulting CNVs were smoothed across five $100 \mathrm{bp}$ windows for a simpler visualization. Final CNVs were then plotted relative to their genomic coordinate at the center of the smoothed window. Since the wild-type CNVs were subtracted from the evolved CNVs, the y axis refers to the copy number change occurred during evolution (i.e. +1 means that one an extra copy of a chromosome fragment has been gained in haploid cells, and that two extra copies have been gained in diploids). Segmental amplifications were identified as continuous chromosomal regions affected by the same change in copy number (Figure 3B and Supplementary table 4). Amplified genes (Figure 5A,C) were obtained from the lists of ORFs included within the boundaries of the segmental amplifications.

\section{The correlation of chromosomal features with segmental amplification boundaries}

We first identified the approximate boundaries ( $\pm 500 \mathrm{bp}$ ) of all segmental amplification by manually inspecting the BAM files with the Integrative Genomic Viewer (https://software.broadinstitute.org/software/igv/). The coordinates of the sequence features that define the boundary between lower and higher read-depths are reported in Supplementary table 4. We then examined the sequences within these approximate boundaries to determine whether various chromosomal features were over- or underrepresented. We considered features that previous studies have found to be associated with hotspots for lesions and sources of genetic instability. We first counted how many times a given feature fell in the boundary zones of segmental amplifications. Then we calculated the expected number of features in these zones based on the total number of features in the genome and the percentage of the genome represented by segmental amplification boundaries zones. We compared these numbers by $\chi 2$ analysis and reported the associated p-values (Supplementary table 7). The number of tRNA genes, transposable elements, LTRs, ARS elements, snRNA and snoRNA genes and centromeres in the genome were determined using YeastMine (https://yeastmine.yeastgenome.org/). Coordinates of fragile sites were obtained from [115]. G4 sequences were obtained from [116]. Highly-(top 5\%) and weakly-(least 5\%) transcribed genes were identified from the data in [117]. Rrm3 binding sites and regions with high levels of $\gamma \mathrm{H} 2 \mathrm{AX}$ were derived from [118] and [119], respectively. Sites of DNA replication termination were derived from [17]. The tandemly repeated sequences, with a minimal repeat tract of twenty-four bases, were obtained from the tandemrepeat-database (TRDB; https://tandem.bu.edu/cgi-bin/trdb/trdb.exe).

\section{DATA AND CODE AVAILABILITY}

A major dataset, containing the sequencing data used in the manuscript has been made publicly available at the EBI European Nucleotide Archive (Accession no: PRJEB42058). The custom pipeline used for the data analysis present in figures and supplementary tables are available on GitHub: https://github.com/marcofumasoni/

\section{ACKNOWLEDGEMENTS}


We thank Michael Laub, María Angélica Bravo Núñez, Sriram Srikant, Thomas LaBar and Andrea Giometto for critical reading of the manuscript; Andrea Giometto for assistance in data analysis; Claire Hartman from the Harvard Bauer Core Facility for technical assistance. We thank the members of the Murray and Nelson labs for helpful discussions. MF acknowledges fellowship support from the Human Frontiers Science Program, EMBO and AIRC.

\section{AUTHOR CONTRIBUTIONS}

MF designed and performed the research, analyzed and interpreted the data, and wrote the paper. AWM designed the research, interpreted the data, and wrote the paper.

\section{BIBLIOGRAPHY}

1. Chandler $\mathrm{CH}$, Chari S, Dworkin I. Does your gene need a background check? How genetic background impacts the analysis of mutations, genes, and evolution. Trends in Genetics. Elsevier Current Trends; 2013. pp. 358-366. doi:10.1016/j.tig.2013.01.009

2. Dowell RD, Ryan O, Jansen A, Cheung D, Agarwala S, Danford T, et al. Genotype to phenotype: A Complex problem. Science. American Association for the Advancement of Science; 2010. p. 469. doi:10.1126/science.1189015

3. Weinreich DM, Lan Y, Jaffe J, Heckendorn RB. The Influence of Higher-Order Epistasis on Biological Fitness Landscape Topography. J Stat Phys. 2018;172: 208-225. doi:10.1007/s10955018-1975-3

4. Fournier T, Schacherer J. Genetic backgrounds and hidden trait complexity in natural populations. Current Opinion in Genetics and Development. Elsevier Ltd; 2017. pp. 48-53. doi:10.1016/j.gde.2017.08.009

5. Filteau M, Hamel V, Pouliot M, Gagnon-Arsenault I, Dubé AK, Landry CR. Evolutionary rescue by compensatory mutations is constrained by genomic and environmental backgrounds. Mol Syst Biol. 2015;11: 832. doi:10.15252/msb.20156444

6. Kempher ML, Tao X, Song R, Wu B, StahI DA, Wall JD, et al. Effects of Genetic and Physiological Divergence on the Evolution of a Sulfate-Reducing Bacterium under Conditions of Elevated Temperature. Rosenzweig RF, Cooper VS, editors. MBio. 2020;11. doi:10.1128/mBio.00569-20

7. Helsen J, Voordeckers K, Vanderwaeren L, Santermans T, Tsontaki M, Verstrepen KJ, et al. Gene loss predictably drives evolutionary adaptation. Mol Biol Evol. 2020. doi:10.1093/molbev/msaa172

8. Harari Y, Ram Y, Rappoport N, Hadany L, Kupiec M. Spontaneous Changes in Ploidy Are Common in Yeast. Curr Biol. 2018;28: 825-835.e4. doi:10.1016/j.cub.2018.01.062

9. Blount ZD, Borland CZ, Lenski RE. Historical contingency and the evolution of a key innovation in an experimental population of Escherichia coli. Proc Natl Acad Sci U S A. 2008;105: 7899-7906. doi:10.1073/pnas.0803151105

10. Jerison ER, Kryazhimskiy S, Mitchell JK, Bloom JS, Kruglyak L, Desai MM. Genetic variation in adaptability and pleiotropy in budding yeast. Elife. 2017;6. doi:10.7554/eLife.27167

11. Rojas Echenique JI, Kryazhimskiy S, Nguyen Ba AN, Desai MM. Modular epistasis and the compensatory evolution of gene deletion mutants. Butler G, editor. PLOS Genet. 2019;15: 
e1007958. doi:10.1371/journal.pgen.1007958

800

801

802

803

804

805

806

807

808

809

810

811

812

813

814

815

816

817

818

819

820

821

822

823

824

825

826

827

828

829

830

831

832

833

834

835

836

12. Szamecz B, Boross G, Kalapis D, Kovács K, Fekete G, Farkas Z, et al. The Genomic Landscape of Compensatory Evolution. Barton NH, editor. PLoS Biol. 2014;12: e1001935. doi:10.1371/journal.pbio.1001935

13. Fumasoni M. Tell Me Where You've Been and I'll Tell You How You'll Evolve. MBio. 2020;11: 2043-2063. doi:10.1128/mBio.02043-20

14. Selmecki AM, Maruvka YE, Richmond PA, Guillet M, Shoresh N, Sorenson AL, et al. Polyploidy can drive rapid adaptation in yeast. Nature. 2015;519: 349-352. doi:10.1038/nature14187

15. Gerstein AC, Cleathero LA, Mandegar MA, Otto SP. Haploids adapt faster than diploids across a range of environments. J Evol Biol. 2011;24: 531-540. doi:10.1111/j.1420-9101.2010.02188.x

16. Paquin C, Adams J. Frequency of fixation of adaptive mutations is higher in evolving diploid than haploid yeast populations. Nature. 1983;302: 495-500. doi:10.1038/302495a0

17. Fumasoni M, Murray AW. The evolutionary plasticity of chromosome metabolism allows adaptation to constitutive DNA replication stress. Elife. 2020;9. doi:10.7554/eLife.51963

18. Zeman MK, Cimprich KA. Causes and consequences of replication stress. Nat Cell Biol. 2014;16: 2-9. doi:10.1038/ncb2897

19. Muñoz S, Méndez J. DNA replication stress: from molecular mechanisms to human disease. Chromosoma. 2016;126: 1-15. doi:10.1007/s00412-016-0573-x

20. Branzei D, Foiani M. Maintaining genome stability at the replication fork. Nat Rev Mol Cell Biol. 2010;11: 208-219. doi:10.1038/nrm2852

21. Bermejo R, Lai MS, Foiani M. Preventing Replication Stress to Maintain Genome Stability: Resolving Conflicts between Replication and Transcription. Mol Cell. 2012;45: 710-718. doi:10.1016/J.MOLCEL.2012.03.001

22. Macheret M, Halazonetis TD. DNA replication stress as a hallmark of cancer. Annu Rev Pathol Mech Dis. 2015;10: 425-48. doi:10.1146/annurev-pathol-012414-040424

23. Di Micco R, Fumagalli M, Cicalese A, Piccinin S, Gasparini P, Luise C, et al. Oncogene-induced senescence is a DNA damage response triggered by DNA hyper-replication. Nature. 2006;444: 638-642. doi:10.1038/nature05327

24. Neelsen KJ, Zanini IMY, Herrador R, Lopes M. Oncogenes induce genotoxic stress by mitotic processing of unusual replication intermediates. J Cell Biol. 2013;200: 699-708. doi:10.1083/jcb.201212058

25. Kotsantis P, Petermann E, Boulton SJ. Mechanisms of oncogene-induced replication stress: Jigsaw falling into place. Cancer Discovery. American Association for Cancer Research Inc.; 2018. pp. 537-555. doi:10.1158/2159-8290.CD-17-1461

26. Weitzman MD, Fradet-Turcotte A. Virus DNA Replication and the Host DNA Damage Response. Annu Rev Virol. 2018;5: 141-164. doi:10.1146/annurev-virology-092917-043534

27. Moody CA. Impact of Replication Stress in Human Papillomavirus Pathogenesis. J Virol. 2018;93. doi:10.1128/jvi.01012-17 
28. Lilley CE, Schwartz RA, Weitzman MD. Using or abusing: viruses and the cellular DNA damage response. Trends in Microbiology. Elsevier; 2007. pp. 119-126. doi:10.1016/j.tim.2007.01.003

29. Scheifele LZ, Cost GJ, Zupancic ML, Caputo EM, Boeke JD. Retrotransposon overdose and genome integrity. Proc Natl Acad Sci U S A. 2009;106: 13927-13932. doi:10.1073/pnas.0906552106

30. San Filippo J, Sung P, Klein H. Mechanism of Eukaryotic Homologous Recombination. Annu Rev Biochem. 2008;77: 229-257. doi:10.1146/annurev.biochem.77.061306.125255

31. Symington LS, Rothstein R, Lisby M. Mechanisms and regulation of mitotic recombination in saccharomyces cerevisiae. Genetics. 2014;198: 795-835. doi:10.1534/genetics.114.166140

32. Powell SN, Kachnic LA. Roles of BRCA1 and BRCA2 in homologous recombination, DNA replication fidelity and the cellular response to ionizing radiation. Oncogene. 2003;22: 5784-5791. doi:10.1038/sj.onc.1206678

33. Walsh CS. Two decades beyond BRCA1/2: Homologous recombination, hereditary cancer risk and a target for ovarian cancer therapy? Gynecologic Oncology. Academic Press Inc.; 2015. pp. 343350. doi:10.1016/j.ygyno.2015.02.017

34. Chernikova SB, Game JC, Brown JM. Inhibiting homologous recombination for cancer therapy. Cancer Biol Ther. 2012;13: 61. doi:10.4161/CBT.13.2.18872

35. Helleday T. Homologous recombination in cancer development, treatment and development of drug resistance. Carcinogenesis. 2010;31: 955-960. doi:10.1093/carcin/bgq064

36. Krajewska M, Fehrmann RSN, De Vries EGE, van Vugt MATM. Regulators of homologous recombination repair as novel targets for cancer treatment. Frontiers in Genetics. Frontiers Media S.A.; 2015. p. 96. doi:10.3389/fgene.2015.00096

37. Villa F, Simon AC, Ortiz Bazan MA, Kilkenny ML, Wirthensohn D, Wightman M, et al. Ctf4 Is a Hub in the Eukaryotic Replisome that Links Multiple CIP-Box Proteins to the CMG Helicase. Mol Cell. 2016;63: 385-96. doi:10.1016/j.molcel.2016.06.009

38. Crabbé L, Thomas A, Pantesco V, De Vos J, Pasero P, Lengronne A. Analysis of replication profiles reveals key role of RFC-Ctf18 in yeast replication stress response. Nat Struct Mol Biol. 2010;17: 1391-1397. doi:10.1038/nsmb.1932

39. Wilhelm T, Olziersky AM, Harry D, De Sousa F, Vassal H, Eskat A, et al. Mild replication stress causes chromosome mis-segregation via premature centriole disengagement. Nat Commun. 2019;10: 1-14. doi:10.1038/s41467-019-11584-0

40. Zheng D-Q, Zhang K, Wu X-C, Mieczkowski PA, Petes TD. Global analysis of genomic instability caused by DNA replication stress in Saccharomyces cerevisiae. Proc Natl Acad Sci U S A. 2016;113: E8114-E8121. doi:10.1073/pnas.1618129113

41. Vesela E, Chroma K, Turi Z, Mistrik M.

Common Chemical Inductors of Replication Stress: Focus on Cell-Based Studies. Biomolecules. 2017;7: 19. doi:10.3390/biom7010019

42. Kouprina NYu, Pashina OB, Nikolaishwili NT, Tsouladze AM, Larionov VL. Genetic control of chromosome stability in the yeast Saccharomyces cerevisiae. Yeast. 1988;4: 257-69. doi:10.1002/yea.320040404 
876

877

878

879

880

881

882

883

884

885

886

887

888

889

890

891

892

893

894

895

896

897

898

899

900

901

902

903

904

905

906

907

908

909

910

911

912

913

914

43. Yuan Z, Georgescu R, Santos R de LA, Zhang D, Bai L, Yao NY, et al. Ctf4 organizes sister replisomes and Pol $\alpha$ into a replication factory. Elife. 2019;8: e47405. doi:10.7554/eLife.47405

44. Samora CP, Saksouk J, Goswami P, Wade BO, Singleton MR, Bates PA, et al. Ctf4 Links DNA Replication with Sister Chromatid Cohesion Establishment by Recruiting the Chl1 Helicase to the Replisome. Mol Cell. 2016;63: 371-84. doi:10.1016/j.molcel.2016.05.036

45. Simon AC, Zhou JC, Perera RL, van Deursen F, Evrin C, Ivanova ME, et al. A Ctf4 trimer couples the CMG helicase to DNA polymerase alpha in the eukaryotic replisome. Nature. 2014/05/09. 2014;510: 293-297. doi:10.1038/nature13234

46. Gambus A, van Deursen F, Polychronopoulos D, Foltman M, Jones RC, Edmondson RD, et al. A key role for $\mathrm{Ctf} 4$ in coupling the MCM2-7 helicase to DNA polymerase alpha within the eukaryotic replisome. EMBO J. 2009/08/08. 2009;28: 2992-3004. doi:10.1038/emboj.2009.226

47. Tanaka H, Katou Y, Yagura M, Saitoh K, Itoh T, Araki H, et al. Ctf4 coordinates the progression of helicase and DNA polymerase alpha. Genes to cells. 2009/06/06. 2009;14: 807-820. doi:10.1111/j.1365-2443.2009.01310.x

48. Abe T, Kawasumi R, Giannattasio M, Dusi S, Yoshimoto Y, Miyata K, et al. AND-1 fork protection function prevents fork resection and is essential for proliferation. Nat Commun. 2018;9: 3091. doi:10.1038/s41467-018-05586-7

49. Fumasoni M, Zwicky K, Vanoli F, Lopes M, Branzei D. Error-Free DNA Damage Tolerance and Sister Chromatid Proximity during DNA Replication Rely on the Pola/Primase/Ctf4 Complex. Mol Cell. 2015;57: 812-823. doi:10.1016/j.molcel.2014.12.038

50. Poli J, Tsaponina O, Crabbé L, Keszthelyi A, Pantesco V, Chabes A, et al. dNTP pools determine fork progression and origin usage under replication stress. EMBO J. 2012;31: 883-894. doi:10.1038/emboj.2011.470

51. Kouprina N, Kroll E, Bannikov V, Bliskovsky V, Gizatullin R, Kirillov A, et al. CTF4 (CHL15) mutants exhibit defective DNA metabolism in the yeast Saccharomyces cerevisiae. Mol Cell Biol. 1992/12/01. 1992;12: 5736-5747. Available: http://www.ncbi.nlm.nih.gov/pubmed/1341195

52. Hanna JS, Kroll ES, Lundblad V, Spencer F a. Saccharomyces cerevisiae CTF18 and CTF4 are required for sister chromatid cohesion. Mol Cell Biol. 2001/04/05. 2001;21: 3144-3158. doi:10.1128/MCB.21.9.3144-3158.2001

53. Pâques F, Haber JE. Multiple pathways of recombination induced by double-strand breaks in Saccharomyces cerevisiae. Microbiol Mol Biol Rev. 1999;63: 349-404. doi: $<p></ p>$

54. Symington LS. Role of RAD52 Epistasis Group Genes in Homologous Recombination and DoubleStrand Break Repair. Microbiol Mol Biol Rev. 2002;66: 630-670. doi:10.1128/mmbr.66.4.630670.2002

55. Lu YB, Ratnakar P V, Mohanty BK, Bastia D. Direct physical interaction between DnaG primase and DnaB helicase of Escherichia coli is necessary for optimal synthesis of primer RNA. Proc Natl Acad Sci USA. 1996;93: 12902-7. doi:10.1073/pnas.93.23.12902

56. Yoshizawa-Sugata N, Masai H. Roles of human AND-1 in chromosome transactions in S phase. J Biol Chem. 2009/05/15. 2009;284: 20718-20728. doi:10.1074/jbc.M806711200 
57. Yeeles JT, Poli J, Marians KJ, Pasero P. Rescuing stalled or damaged replication forks. Cold Spring Harb Perspect Biol. 2013/05/03. 2013;5: a012815. doi:10.1101/cshperspect.a012815

58. Gerrish PJ, Lenski RE. The fate of competing beneficial mutations in an asexual population.

59. Lang GI, Botstein D, Desai MM. Genetic variation and the fate of beneficial mutations in asexual populations. Genetics. 2011;188: 647-661. doi:10.1534/genetics.111.128942

60. Venkataram S, Dunn B, Li Y, Agarwala A, Chang J, Ebel ER, et al. Development of a Comprehensive Genotype-to-Fitness Map of Adaptation-Driving Mutations in Yeast. Cell. 2016;166: 15851596.e22. doi:10.1016/J.CELL.2016.08.002

61. Gerstein AC, Chun H-JE, Grant A, Otto SP. Genomic Convergence toward Diploidy in Saccharomyces cerevisiae. Dutcher S, editor. PLoS Genet. 2006;2: e145. doi:10.1371/journal.pgen.0020145

62. Gerstein AC, Otto SP. Cryptic fitness advantage: Diploids invade haploid populations despite lacking any apparent advantage as measured by standard fitness assays. PLoS One. 2011;6: 26599. doi:10.1371/journal.pone.0026599

63. Couce A, Tenaillon OA. The rule of declining adaptability in microbial evolution experiments. Front Genet. 2015;6: 99. doi:10.3389/fgene.2015.00099

64. Wiser JM, Ribeck N, Lenski RE. Long-Term Dynamics of Adaptation in Asexual Populations. Science (80- ). 2013;342: 1364-1367. doi:10.1126/science.1243357

65. Chou H, Chiu H, Delaney NF, Segrè D, Marx CJ. Diminishing Returns Epistasis Among Beneficial Mutations Decelerates Adaptation. Science (80- ). 2011;332: 1190-1193. doi:10.1126/science.1203799

66. Khan Al, Dinh DM, Schneider D, Lenski RE, Cooper TF. Negative epistasis between beneficial mutations in an evolving bacterial population. Science (80- ). 2011;332: 1193-1196. doi:10.1126/science.1203801

67. Barrick JE, Kauth MR, Strelioff CC, Lenski RE. Escherichia coli rpoB mutants have increased evolvability in proportion to their fitness defects. Mol Biol Evol. 2010;27: 1338-1347. doi:10.1093/molbev/msq024

68. Kryazhimskiy S, Rice DP, Jerison ER, Desai MM. Microbial evolution. Global epistasis makes adaptation predictable despite sequence-level stochasticity. Science. 2014;344: 1519-1522. doi:10.1126/science.1250939

69. Marad DA, Buskirk SW, Lang Gl. Altered access to beneficial mutations slows adaptation and biases fixed mutations in diploids. Nat Ecol Evol. 2018;2: 882-889. doi:10.1038/s41559-0180503-9

70. Zeyl C, Vanderford T, Carter M. An Evolutionary Advantage of Haploidy in Large Yeast Populations. Science (80- ). 2003;299: 555-558. doi:10.1126/SCIENCE.1078417

71. Von Borstel RC, Cain KT, Steinberg CM. Inheritance of spontaneous mutability in yeast. Genetics. 1971;69: 17-27. Available: https://www.ncbi.nlm.nih.gov/pmc/articles/PMC1212686/

72. Kunz BA, Peters MG, Kohalmi SE, Armstrong JD, Glattke M, Badiani K. Disruption of the RAD52 
gene alters the spectrum of spontaneous SUP4-o mutations in Saccharomyces cerevisiae. Genetics. 1989;122: 535-542. Available: /pmc/articles/PMC1203727/?report=abstract

73. Puddu F, Herzog M, Selivanova A, Wang S, Zhu J, Klein-Lavi S, et al. Genome architecture and stability in the Saccharomyces cerevisiae knockout collection. Nature. 2019; 1-5. doi:10.1038/s41586-019-1549-9

74. Rolef Ben-Shahar T, Heeger S, Lehane C, East P, Flynn H, Skehel M, et al. Eco1-dependent cohesin acetylation during establishment of sister chromatid cohesion. Science (80- ). 2008/07/26. 2008;321: 563-566. doi:10.1126/science.1157774

75. Panizza S, Tanaka T, Hochwagen A, Eisenhaber F, Nasmyth K. Pds5 cooperates with cohesion in maintaining sister chromatid cohesion. Curr Biol. 2000;10: 1557-1564. doi:10.1016/S09609822(00)00854-X

76. Michaelis C, Ciosk R, Nasmyth K. Cohesins: chromosomal proteins that prevent premature separation of sister chromatids. Cell. 1997/10/23. 1997;91: 35-45. doi:doi: 10.1016/s00928674(01)80007-6.

77. Ciosk R, Shirayama M, Shevchenko A, Tanaka T, Toth A, Nasmyth K. Cohesin's binding to chromosomes depends on a separate complex consisting of Scc2 and Scc4 proteins. Mol Cell. 2000/07/06. 2000;5: 243-254. doi:10.1016/S1097-2765(00)80420-7

78. Srinivasan M, Fumasoni M, Petela NJ, Murray A, Nasmyth KA. Cohesion is established during DNA replication utilising chromosome associated cohesin rings as well as those loaded de novo onto nascent DNAs. Elife. 2020;9. doi:10.7554/eLife.56611

79. St. Charles J, Petes TD. High-Resolution Mapping of Spontaneous Mitotic Recombination Hotspots on the 1.1 Mb Arm of Yeast Chromosome IV. Klein H, editor. PLoS Genet. 2013;9: e1003434. doi:10.1371/journal.pgen.1003434

80. Sharp NP, Sandell L, James CG, Otto SP. The genome-wide rate and spectrum of spontaneous mutations differ between haploid and diploid yeast. Proc Natl Acad Sci USA. 2018;115: E5046E5055. doi:10.1073/pnas.1801040115

81. Serero A, Jubin C, Loeillet S, Legoix-Né P, Nicolas AG. Mutational landscape of yeast mutator strains. Proc Natl Acad Sci U S A. 2014;111: 1897-1902. doi:10.1073/pnas.1314423111

82. Loeillet S, Herzog M, Puddu F, Legoix P, Baulande S, Jackson SP, et al. Trajectory and uniqueness of mutational signatures in yeast mutators. Proc Natl Acad Sci U S A. 2020;117: 24947-24956. doi:10.1073/pnas.2011332117

83. Fisher KJ, Buskirk SW, Vignogna RC, Marad DA, Lang GI. Adaptive genome duplication affects patterns of molecular evolution in Saccharomyces cerevisiae. Otto SP, editor. PLOS Genet. 2018;14: e1007396. doi:10.1371/journal.pgen.1007396

84. Scott AL, Richmond PA, Dowell RD, Selmecki AM. The Influence of Polyploidy on the Evolution of Yeast Grown in a Sub-Optimal Carbon Source. Mol Biol Evol. 2017;34: 2690-2703. doi:10.1093/molbev/msx205

85. Dunham MJ, Badrane H, Ferea T, Adams J, Brown PO, Rosenzweig F, et al. Characteristic genome rearrangements in experimental evolution of Saccharomyces cerevisiae. Proc Natl Acad Sci U S A. 2002;99: 16144-16149. doi:10.1073/pnas.242624799 
86. Gresham D, Desai MM, Tucker CM, Jenq HT, Pai DA, Ward A, et al. The Repertoire and Dynamics of Evolutionary Adaptations to Controlled Nutrient-Limited Environments in Yeast. Snyder M, editor. PLoS Genet. 2008;4: e1000303. doi:10.1371/journal.pgen.1000303

87. Lauer S, Avecilla G, Spealman P, Sethia G, Brandt N, Levy SF, et al. Single-cell copy number variant detection reveals the dynamics and diversity of adaptation. de Visser JAGM, editor. PLOS Biol. 2018;16: e3000069. doi:10.1371/journal.pbio.3000069

88. Tsaponina O, Barsoum E, Åström SU, Chabes A. Ixr1 is required for the expression of the ribonucleotide reductase Rnr1 and maintenance of dNTP pools. PLoS Genet. 2011;7. doi:10.1371/journal.pgen.1002061

89. Takayama Y, Kamimura Y, Okawa M, Muramatsu S, Sugino A, Araki H. GINS, a novel multiprotein complex required for chromosomal DNA replication in budding yeast. Genes Dev. 2003;17: 11531165. doi:10.1101/gad.1065903

90. Orr HA, Otto SP. Does diploidy increase the rate of adaptation? Genetics. 1994;136: 1475-1480. Available: /pmc/articles/PMC1205926/?report=abstract

91. Tlsty TD, Albertini AM, Miller JH. Gene amplification in the lac region of E. coli. Cell. 1984;37: 217-224. doi:10.1016/0092-8674(84)90317-9

92. Slack A, Thornton PC, Magner DB, Rosenberg SM, Hastings PJ. On the Mechanism of Gene Amplification Induced under Stress in Escherichia coli. Matic I, editor. PLoS Genet. 2006;2: e48. doi:10.1371/journal.pgen.0020048

93. Reddy G, Desai MM. Global epistasis emerges from a generic model of a complex trait. bioRxiv. 2020; 2020.06.14.150946. doi:10.1101/2020.06.14.150946

94. Wünsche A, Dinh DM, Satterwhite RS, Arenas CD, Stoebel DM, Cooper TF. Diminishing-returns epistasis decreases adaptability along an evolutionary trajectory. Nat Ecol Evol. 2017;1: 0061. doi:10.1038/s41559-016-0061

95. Schoustra S, Hwang S, Krug J, de Visser JAGM. Diminishing-returns epistasis among random beneficial mutations in a multicellular fungus. Proc R Soc B Biol Sci. 2016;283: 20161376. doi:10.1098/rspb.2016.1376

96. Lyons DM, Zou Z, Xu H, Zhang J. Idiosyncratic epistasis creates universals in mutational effects and evolutionary trajectories. Nat Ecol Evol. 2020;4: 1685-1693. doi:10.1038/s41559-020-01286y

97. Deutschbauer AM, Jaramillo DF, Proctor M, Kumm J, Hillenmeyer ME, Davis RW, et al. Mechanisms of Haploinsufficiency Revealed by Genome-Wide Profiling in Yeast. Genetics. 2005;169: 1915-1925. doi:10.1534/GENETICS.104.036871

98. Gerstein AC, Otto SP. Ploidy and the Causes of Genomic Evolution. J Hered. 2009;100: 571-581. doi:10.1093/jhered/esp057

99. Otto SP, Gerstein AC. The evolution of haploidy and diploidy. Current Biology. Elsevier; 2008. pp. R1121-R1124. doi:10.1016/j.cub.2008.09.039

100. Schacherer J, de Montigny J, Welcker A, Souciet JL, Potier S. Duplication processes in Saccharomyces cerevisiae haploid strains. Nucleic Acids Res. 2005;33: 6319-6326. 
1033

1034

1035

1036

1037

1038

1039

1040

1041

1042

1043

1044

1045

1046

1047

1048

1049

1050

1051

1052

1053

1054

1055

1056

1057

1058

1059

1060

1061

1062

1063

1064

1065

1066

1067

1068

1069

1070

1071

doi:10.1093/nar/gki941

101. Coïc E, Feldman T, Landman AS, Haber JE. Mechanisms of Rad52-independent spontaneous and UV-induced mitotic recombination in Saccharomyces cerevisiae. Genetics. 2008;179: 199-211. doi:10.1534/genetics.108.087189

102. Prado F, González-Barrera S, Aguilera A. RAD52-dependent and -independent homologous recombination initiated by Flp recombinase at a single FRT site flanked by direct repeats. Mol Gen Genet. 2000;263: 73-80. doi:10.1007/PL00008677

103. Payen C, Koszul R, Dujon B, Fischer G. Segmental Duplications Arise from Pol32-Dependent Repair of Broken Forks through Two Alternative Replication-Based Mechanisms. Haber JE, editor. PLoS Genet. 2008;4: e1000175. doi:10.1371/journal.pgen.1000175

104. Naiman K, Pagès V, Fuchs RP. A defect in homologous recombination leads to increased translesion synthesis in E. coli. Nucleic Acids Res. 2016;44: 7691-7699. doi:10.1093/nar/gkw488

105. LaBar T, Phoebe Hsieh Y-Y, Fumasoni M, Murray AW. Evolutionary Repair Experiments as a Window to the Molecular Diversity of Life. Curr Biol. 2020;30: R565-R574. doi:10.1016/J.CUB.2020.03.046

106. Russo M, Crisafulli G, Sogari A, Reilly NM, Arena S, Lamba S, et al. Adaptive mutability of colorectal cancers in response to targeted therapies. Science (80- ). 2019;366: 1473-1480. doi:10.1126/science.aav4474

107. Thiagalingam S, Laken S, Willson JKV, Markowitz SD, Kinzler KW, Vogelstein B, et al. Mechanisms underlying losses of heterozygosity in human colorectal cancers. Proc Natl Acad Sci U S A. 2001;98: 2698-2702. doi:10.1073/pnas.051625398

108. D'Angiolo M, Chiara M De, Yue J-X, Irizar A, Stenberg S, Persson K, et al. A yeast living ancestor reveals the origin of genomic introgressions. Nature. 2020; $1-6$. doi:10.1038/s41586-020-2889-1

109. Peter J, De Chiara M, Friedrich A, Yue J-X, Pflieger D, Bergström A, et al. Genome evolution across 1,011 Saccharomyces cerevisiae isolates. Nature. 2018;556: 339-344. doi:10.1038/s41586-0180030-5

110. Steenwyk JL, Opulente DA, Kominek J, Shen X-X, Zhou X, Labella AL, et al. Extensive loss of cellcycle and DNA repair genes in an ancient lineage of bipolar budding yeasts. Kamoun $S$, editor. PLOS Biol. 2019;17: e3000255. doi:10.1371/journal.pbio.3000255

111. Ghosal G, Chen J. DNA damage tolerance: a double-edged sword guarding the genome. Translational Cancer Research. 2013. pp. 107-129. doi:10.3978/j.issn.2218-676X.2013.04.01

112. Hohl M, Mojumdar A, Hailemariam S, Kuryavyi V, Ghisays F, Sorenson K, et al. Modeling cancer genomic data in yeast reveals selection against ATM function during tumorigenesis. Symington LS, editor. PLOS Genet. 2020;16: e1008422. doi:10.1371/journal.pgen.1008422

113. Koboldt DC, Zhang $Q$, Larson DE, Shen D, McLellan MD, Lin L, et al. VarScan 2: somatic mutation and copy number alteration discovery in cancer by exome sequencing. Genome Res. 2012;22: 568-76. doi:10.1101/gr.129684.111

114. Franceschini A, Szklarczyk D, Frankild S, Kuhn M, Simonovic M, Roth A, et al. STRING v9.1: Protein-protein interaction networks, with increased coverage and integration. Nucleic Acids Res. 
1073

1074

1075

1076

1077

1078

1079

1080

1081

1082

1083

1084

1085

1086

1087

1088
115. Song W, Dominska M, Greenwell PW, Petes TD. Genome-wide high-resolution mapping of chromosome fragile sites in Saccharomyces cerevisiae. Proc Natl Acad Sci U S A. 2014;111: E2210-8. doi:10.1073/pnas.1406847111

116. Capra JA, Paeschke K, Singh M, Zakian VA. G-Quadruplex DNA Sequences Are Evolutionarily Conserved and Associated with Distinct Genomic Features in Saccharomyces cerevisiae. Stormo GD, editor. PLoS Comput Biol. 2010;6: e1000861. doi:10.1371/journal.pcbi.1000861

117. Nagalakshmi U, Wang Z, Waern K, Shou C, Raha D, Gerstein M, et al. The Transcriptional Landscape of the Yeast Genome Defined by RNA Sequencing. Science (80- ). 2008;320: 13441350.

118. Azvolinsky A, Dunaway S, Torres JZ, Bessler JB, Zakian VA. The S. cerevisiae Rrm3p DNA helicase moves with the replication fork and affects replication of all yeast chromosomes. Genes Dev. 2006;20: 3104-16. doi:10.1101/gad.1478906

119. Szilard RK, Jacques P-E, Laramée L, Cheng B, Galicia S, Bataille AR, et al. Systematic identification of fragile sites via genome-wide location analysis of gamma-H2AX. Nat Struct Mol Biol. 2010;17: 299-305. doi:10.1038/nsmb.1754 